\title{
TITLE:
}

\section{Chaotic mixing and mixing efficiency in a short time}

\author{
$\operatorname{AUTHOR}(\mathrm{S})$ : \\ Funakoshi, Mitsuaki
}

\section{CITATION:}

Funakoshi, Mitsuaki. Chaotic mixing and mixing efficiency in a short time. Fluid Dynamics Research 2008, 40(1): 1-33

\section{ISSUE DATE:}

2008-01

URL:

http://hdl.handle.net/2433/254160

\section{RIGHT:}

This is an author-created, un-copyedited version of an article accepted for publication in 'Fluid Dynamics Research'. The publisher is not responsible for any errors or omissions in this version of the manuscript or any version derived from it. The Version of Record is available online at https://doi.org/10.1016/j.fluiddyn.2007.04.004.; The full-text file will be made open to the public on 1 January 2009 in accordance with publisher's 'Terms and Conditions for Self-Archiving'.; This is not the published version. Please cite only the published version.; この論文は出版社版でありません。引用の際 には出版社版をご確認ご利用ください。 


\title{
Chaotic Mixing and Mixing Efficiency in a Short Time
}

\author{
Mitsuaki FUNAKOSHI \\ Department of Applied Analysis and Complex Dynamical Systems, \\ Graduate School of Informatics, Kyoto University, \\ Yoshida-Honmachi, Sakyo-ku, Kyoto, 606-8501
}

\begin{abstract}
Several studies of the chaotic motion of fluid particles by two-dimensional timeperiodic flows or three-dimensional steady flows, called Lagrangian chaos, are first introduced. Secondly, some of the studies on efficient mixing caused by Lagrangian chaos, called chaotic mixing, are reviewed with discussion of several indices for the estimation of mixing efficiency. Finally, several indices to estimate the efficiency of mixing in a short time, such as those related to transport matrices, stable and unstable manifolds of hyperbolic periodic points of Poincaré maps, and lines of separation, are explained by showing examples of mixing by two-dimensional time-periodic flows between eccentric rotating cylinders and mixing by three-dimensional steady flows in a model of static mixers.
\end{abstract}

key words : chaotic mixing, Lagrangian chaos, transport matrix, stable and unstable manifolds.

\section{§1. Introduction}

Mixing of fluids is one of the important subjects in fluid dynamics, and has been studied for many years. When we consider the mixing of two incompressible miscible fluids $\mathrm{A}$ and $\mathrm{B}$ in a container initially occupying two disjoint regions of volumes $V_{A}$ and $V_{B}$ respectively by a flow of these fluids, our goal is to attain a completely mixed state in which the volume ratio $r_{A}$ of fluid $\mathrm{A}$ in any region is equal to $\bar{r}_{A}=V_{A} /\left(V_{A}+V_{B}\right)$. If there is no diffusion between the fluids, the value of $r_{A}$ in a sufficiently small region can be far from $\bar{r}_{A}$ even after a long-time motion of fluid elements by the flow, although the boundary between these fluids may be quite complicated. However, since a diffusive 
effect actually exists between miscible fluids, if a striped pattern of the fluids with a sufficiently small spacing is attained by the flow, a rapid approach to the completely mixed state is expected because of the large gradient of a concentration of each fluid in this pattern. Therefore, when we examine the mixing of fluids caused by a flow in the absence of a diffusive effect, it is sufficient to aim at attaining the approximately mixed state in which the value of $r_{A}$ within any region of size larger than $\delta$ is close to $\bar{r}_{A}$. The value of $\delta$ is determined by the time provided for mixing and the value of a diffusion constant.

The efficiency of mixing by a turbulent flow is expected to be much higher than that by a laminar flow, because a small-scale and high-frequency motion in a turbulent flow has a diffusive effect on the mixing in a large scale. However, mixing by a laminar flow is important in a few cases. That is, if large amounts of fluids of high viscosity are to be mixed in chemical plants, glass production systems, and so on, these fluids are sometimes mixed by a laminar flow because it is inefficient to generate a turbulent flow using a large amount of power. Next, mixing of fluids is now important also in fluid systems of dimensions ranging from micrometers to millimeters because of the development in microscale devices and analyzers. Since the Reynolds number characterizing the flows in such systems is usually low, fluids are mixed by a laminar flow in most cases. The mixing at microscale was reviewed by Stremler et al. (2004) and by Ottino and Wiggins (2004). Reviews on micromixers were also written by Hessel et al. (2005), by Chew et al. (2005), and by Dittrich et al. (2006). Moreover, in some food production systems, fluid materials have to be mixed gently or slowly by a laminar flow so that their fine structure is not destroyed. Furthermore, when we examine the mixing or transport of materials in the atmosphere or ocean, such as pollutants, volcanic gas and ashes, by large-scale flows, these flows are often modeled as laminar with the introduction of eddy viscosity that is much larger than molecular viscosity.

In mixing by laminar flows, the attainment of a striped pattern with a smaller spacing is required, compared with the case of mixing by turbulent flows, because there is only a weak diffusive effect associated with molecular viscosity. Therefore, careful and severe estimation of each flow on how fast this flow can generate a uniform striped pattern with a sufficiently small spacing is important in the study of mixing by such flows. 
Taking this into account, in the present paper, we consider the mixing of fluids caused by laminar flows under the assumption of no diffusive effect. The mixing of fluids by a steady or time-periodic flow can be related to the theory of dynamical systems if this flow is independent of the configuration of fluids. Moreover, the chaotic motion of fluid particles caused by a flow, if it is observed, is expected to yield efficient mixing. Therefore many studies of the chaotic motion of fluid particles, called Lagrangian chaos, and of the efficient mixing of fluids by utilizing this chaotic motion, called chaotic mixing, have developed from the 1980's.

In Section 2 of the present paper, after describing the relation of a few basic concepts in the theory of dynamical systems and in the theory of the application of chaos to fluid dynamics, we give a brief summary of preceding studies of Lagrangian chaos. In Section 3 , we describe a few results on the efficiency of mixing obtained from the above theories. We also introduce several indices of mixing efficiency that are often used in the study of chaotic mixing, and attempt to summarize some of the many studies on chaotic mixing caused by two-dimensional time-periodic flows or three-dimensional steady flows.

Some of the indices of mixing efficiency suggested or used in these studies are explicitly or implicitly related to the efficiency of mixing over a long time. However, in the applications described above, it is sometimes difficult or impossible to carry out the mixing of fluids for a long time. Therefore, the indices that can be used to estimate the mixing efficiency in a short time may also be important. In Section 4, some results on the indices of efficiency of mixing in a short time are shown, with special attention to mixing by two-dimensional time-periodic flows between eccentric rotating cylinders and by three-dimensional steady flows in a model of a static mixer. Section 5 is devoted to concluding remarks.

\section{$\S 2$. Lagrangian chaos}

If velocity field $\boldsymbol{u}(\boldsymbol{x}, t)$ of a laminar flow is given as a function of spatial coordinate $\boldsymbol{x}$ and time $t$, the position $\boldsymbol{X}(t)$ of a fluid particle in this flow evolves according to the following equation:

$$
\frac{d \boldsymbol{X}(t)}{d t}=\boldsymbol{u}(\boldsymbol{X}(t), t)
$$

This equation is a dynamical system for two (three) dependent variables if we consider 
the motion of a fluid particle by a two-dimensional (three-dimensional) flow, and is an autonomous (non-autonomous) system for a steady (unsteady) flow. It is widely known that chaotic solutions characterized by irregular time evolution and sensitive dependence on initial conditions are possible only in autonomous systems for three or more variables, or in non-autonomous systems for two or more variables. Therefore, although the chaotic motion of fluid particles is not allowed in a steady two-dimensional flow, their motion can be chaotic in a three-dimensional steady flow or in a two-dimensional time-periodic flow. This chaotic motion is often called Lagrangian chaos, because $\boldsymbol{X}(t)$ is one of the dependent variables in the Lagrangian description of fluid motions. Since the dependent variable $\boldsymbol{X}(t)$ of Eq. (1) is in physical space, if this chaos exists, we can directly observe characteristic geometrical properties of chaos such as the stretching and folding of a set of initial points in numerical or experimental studies on Lagrangian chaos by using computer graphics or flow visualization techniques. Although Lagrangian chaos is possible in more complicated flows such as three-dimensional time-periodic flows and two-dimensional quasi-periodic flows, most of the studies of Lagrangian chaos up to now are for two-dimensional time-periodic flows or three-dimensional steady flows, probably because useful information is not obtained easily from the application of the theory of dynamical systems for more complicated flows.

Moreover, in most of the studies of Lagrangian chaos, the motion of fluid particles by flows of an incompressible fluid is examined. The velocity field $\boldsymbol{u}(\boldsymbol{x}, t)$ of this fluid must satisfy the following equation of continuity:

$$
\operatorname{div} \boldsymbol{u}=0
$$

When Eq. (2) is satisfied, Eq. (1) is a conservative system, in which the volume of any region in the space of dependent variables is conserved when each point within this region moves according to this system. This property directly corresponds to the incompressibility of the fluid. For the special case of a two-dimensional flow of an incompressible fluid in the $(x, y)$ plane, since $u$ and $v$, the $x$ and $y$ components of $\boldsymbol{u}$, satisfy Eq. (2), we can introduce a streamfunction $\psi$ satisfying $u=\partial \psi / \partial y$ and $v=-\partial \psi / \partial x$. Using this $\psi$, Eq. (1) is expressed as

$$
\frac{d X}{d t}=\frac{\partial \psi(X, Y, t)}{\partial Y}, \frac{d Y}{d t}=-\frac{\partial \psi(X, Y, t)}{\partial X}
$$


where $(X, Y)$ are the $(x, y)$ components of $\boldsymbol{X}$. Equation (3) is the canonical equation of a one-degree-of-freedom Hamiltonian system with Hamiltonian $\psi$.

Conservative systems are classified into integrable and non-integrable systems. The $n$-degree-of-freedom Hamiltonian systems, which are typical examples of conservative systems, are integrable only if they have $n$ independent conserved quantities. Chaotic solutions are known to be possible only in non-integrable systems. Therefore, the motion of fluid particles by a three-dimensional steady flow can be chaotic because Eq. (1) can be non-integrable. Moreover, for a two-dimensional time-periodic flow, the streamfunction $\psi$ in Eq. (3) explicitly depends on $t$ and then is no longer conserved. Therefore, the motion of fluid particles by this flow also can be chaotic since Eq. (3) can be non-integrable.

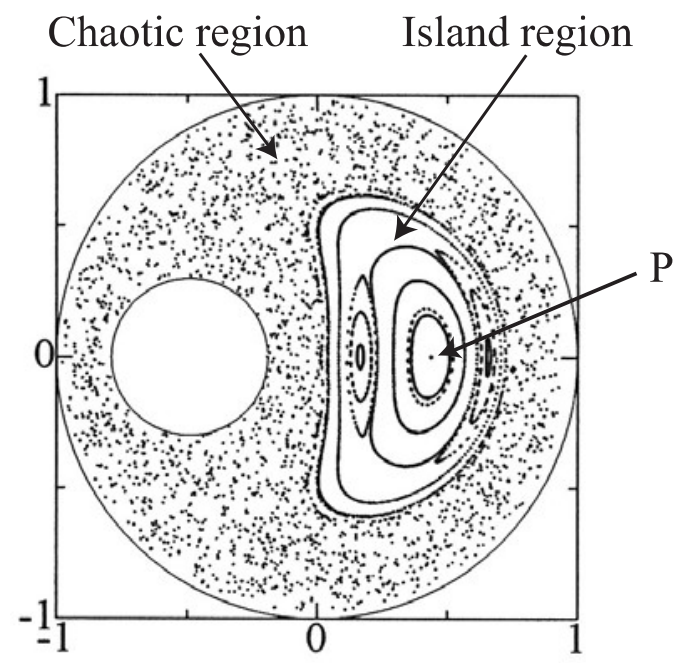

Figure 1: An example of a Poincaré section. $\alpha=0.3, \varepsilon=0.7, T_{\text {out }}=T_{\text {in }}=1.0$. The definition of these parameters is given in Section 4.

When we consider the motion of fluid particles by a two-dimensional time-periodic flow with period $T$, we can define a two-dimensional area-preserving map $M$ that maps $\boldsymbol{X}(t)=(X(t), Y(t))$ onto $\boldsymbol{X}(t+T)=(X(t+T), Y(t+T))$. This map is called a Poincaré map. Furthermore, by plotting the locations $\boldsymbol{X}(n T)=M^{n}(\boldsymbol{X}(0))$ of a few fluid particles starting from initial positions $\boldsymbol{X}(0)$ for many values of the integer $n=0,1,2, \ldots$, we obtain a figure composed of many points on the $(X, Y)$ plane. This figure is called a Poincaré section. Figure 1 shows an example of Poincaré section produced from the 
motions of 20 fluid particles for $n=0,1, \ldots, 300$ by the two-dimensional slow timeperiodic flow between two eccentric cylinders that rotate alternately by $2 \pi$ radian in the same direction in each period. The details of this flow will be described in Section 4.

Since the Poincaré map $M$ is area-preserving, the periodic points (including fixed points of period one) of this map are hyperbolic (saddle type), elliptic (center type), or parabolic, depending on the eigenvalues of Jacobian matrix associated with these periodic points, as explained below. Since $M$ is a two-dimensional map, the relation $\boldsymbol{X}\left(t_{0}+p T\right)=M^{p}\left(\boldsymbol{X}\left(t_{0}\right)\right)$ for any positive integer $p$ can be expressed as

$$
z_{1}\left(t_{0}+p T\right)=M_{1}^{(p)}\left(z_{1}\left(t_{0}\right), z_{2}\left(t_{0}\right)\right), \quad z_{2}\left(t_{0}+p T\right)=M_{2}^{(p)}\left(z_{1}\left(t_{0}\right), z_{2}\left(t_{0}\right)\right),
$$

where $z_{1}$ and $z_{2}$ are $X$ and $Y$, respectively, and $t_{0}$ is a constant. Moreover, if $\boldsymbol{X}^{(p)}=$ $\left(X^{(p)}, Y^{(p)}\right)$ is one of the periodic points of $M$ of period $p$, it satisfies

$$
M^{p}\left(\boldsymbol{X}^{(p)}\right)=\boldsymbol{X}^{(p)}, \quad M^{q}\left(\boldsymbol{X}^{(p)}\right) \neq \boldsymbol{X}^{(p)}, \text { for } q=1,2, \ldots, p-1 .
$$

The $(i, j)$ component of the Jacobian matrix $G$ of map $M^{p}$ associated with a periodic point $\boldsymbol{X}^{(p)}$ is written as

$$
\left(\frac{\partial M_{i}^{(p)}}{\partial z_{j}}\right)_{z_{1}=X^{(p)}, z_{2}=Y^{(p)}}, \quad(i, j=1,2) .
$$

Since $M$ is area-preserving, the two eigenvalues $\mu_{1}$ and $\mu_{2}$ of $G$ satisfy $\mu_{1} \mu_{2}=1$. If the imaginary parts of $\mu_{1}$ and $\mu_{2}$ satisfying $\mu_{2}=\mu_{1}^{*}$ are non-zero and $\left|\mu_{1}\right|=\left|\mu_{2}\right|=$ 1 is satisfied, $\boldsymbol{X}^{(p)}$ is neutrally stable and is called elliptic, where $*$ denotes complex conjugate. However, if both $\mu_{1}$ and $\mu_{2}$ are real, and satisfy the relation $\left|\mu_{1}\right|>1>\left|\mu_{2}\right|$, $\boldsymbol{X}^{(p)}$ is unstable and is called hyperbolic. Moreover, if both $\mu_{1}$ and $\mu_{2}$ are equal to $1, \boldsymbol{X}^{(p)}$ is called parabolic. Parabolic periodic points are usually observed for the parameters of flows where the type of these points changes from elliptic to hyperbolic. In the following part, $\mu_{1}$ is assumed to satisfy the condition $\left|\mu_{1}\right|>1$ for hyperbolic periodic points, and is called an unstable eigenvalue.

In Poincaré sections, if $\boldsymbol{X}(0)$ is sufficiently close to one of the elliptic periodic points of period $p$, the points $\boldsymbol{X}(n p T)$ are on one of the closed curves encircling this point for all integer $n$. An example of this behavior is shown in Fig. 1 by a few closed curves encircling 
the elliptic fixed point $\mathrm{P}$ on the right-hand side of the inner cylinder. The region around elliptic periodic points of period $p$ where the positions $\boldsymbol{X}(n p T)$ of fluid particles starting from this region move regularly on one of the curves encircling these points is called an island region. In Fig. 1, we observe a typical island region around P. Near the two cylinders of this figure, there are also regions where $\boldsymbol{X}(n T)$ moves regularly on a closed curve along either of the cylinders, similarly to that in island regions, although their widths are too small to distinguish. These regions around elliptic periodic points or near the boundaries of the fluid region are called regular regions because fluid particles starting from these regions move regularly.

In Poincaré sections, it is also commonly observed that the location $\boldsymbol{X}(n T)$ of fluid particles starting from a certain region where many points are scattered randomly varies irregularly within this region. This region is called a chaotic region because we observe irregular movement and sensitive dependence on initial conditions of $\boldsymbol{X}(n T)$ starting from this region. In Fig. 1, this region occupies a relatively large part of the fluid region between two eccentric cylinders.

Therefore, reflecting the characteristic property of solutions to conservative systems, the motion of fluid particles is chaotic or regular depending on their initial locations. Here the boundaries between regular and chaotic regions usually have a fractal structure. That is, the boundaries include a fine complicated geometrical structure that is repeatedly observed after every specified magnification of the region around the boundaries. Detailed descriptions of fractals were given by Mandelbrot (1982). Moreover, some small island regions may be embedded in chaotic regions. Furthermore, fluid particles can move chaotically within thin layers satisfying a resonant condition included in regular regions. However, using Poincaré sections, we can roughly divide the region occupied by a fluid into regular and chaotic regions, although rigorous division is difficult.

Since the 1980's, there have been many studies of Lagrangian chaos in two-dimensional time-periodic flows. These studies were carried out by experiments, by numerical simulations, by theoretical analysis, or by the combination of these methods. Among these studies, the Lagrangian chaos induced by the slow time-periodic flow between eccentric rotating cylinders introduced in Fig. 1 was examined most extensively, probably because this flow field not only is realized easily in experiments but also is expressed in an ana- 
lytic form. This flow was generalized without losing these preferable characteristics by Finn and Cox (2001) so that translational motions of the inner cylinder are also allowed.

Some of the other studies based on theoretical analysis or numerical simulations used quite simple flows such as the flow induced by blinking point vortices (Aref, 1984) and the flow by a pulsed source-sink system (Jones and Aref, 1988) so that the appearance and development of Lagrangian chaos with the variation in flow parameters can be examined easily, although it is difficult to produce such flows in experiments. On the other hand, several experimental or numerical studies have been performed in which the flows generated by periodic motions of simple flow boundaries were used. Unfortunately, however, most of the velocity fields corresponding to these flows are obtained only numerically. This sometimes demands a careful interpolation of velocity data if these data are given only at discrete points in order to obtain acceptable trajectories of fluid particles after a long time. A typical example of such flows is the slow time-periodic flow in a lid-driven cavity generated by the alternate periodic motion of upper and lower boundaries parallel to themselves (see, for example, Leong and Ottino, 1989 and Franjione et al. 1989).

An interesting subject related to Lagrangian chaos by two-dimensional flows is topological chaos. This chaos, first examined by Boyland et al. (2000), is produced by an appropriate slow motion of three or more stirring rods in a container (batch mixer). This motion is determined on the basis of topology and braid theory. A recent review of this subject was written by Thiffeault and Finn (2006).

Studies of Lagrangian chaos in three-dimensional steady flows also have been performed intensively, with stronger interest in its relation to actual mixing devices. Since Eq. (1) governing the motion of fluid particles is a conservative system, the region of a fluid can be roughly divided into regular and chaotic regions, similarly to the case of two-dimensional time-periodic flows. Within regular regions, each fluid particle moves regularly on a closed curve or on a torus surrounding it if the fluid region is bounded. Moreover, if the steady flows are spatially periodic in an unbounded fluid region, as in the case of static mixers, each fluid particle in regular regions moves regularly along an unbounded periodic curve or along a surface of an unbounded periodic tubular region surrounding this curve. Here static mixers are tubular mixing devices that include periodic elements of axial period $L$ to generate a cross-sectional flow, from one end of 
which fluids to be mixed are fed by imposing a high pressure, and at the other end of which the outflow of a mixed fluid is expected. Therefore, the above curve and tubular region extend along the axis of this mixer with period $L$. On the other hand, the motion of fluid particles in chaotic regions is irregular, and their trajectories are geometrically complicated. Since these trajectories are the same as streamlines, Lagrangian chaos in three-dimensional steady flows is also called chaos of streamlines. When chaotic regions are dominant, regular regions composed of thin tori or tubes are surrounded by the chaotic regions.

In order to capture the characteristics of motions of fluid particles in a three-dimensional fluid region, it is better to examine the intersection of their trajectories with a plane. Therefore, if this fluid region is bounded, we often try to find a plane such that most of fluid particles in this region pass through this plane and return to it many times. If such a plane is obtained, we can define the Poincaré map $M$ that maps the initial position of a fluid particle on this plane onto its location at the time of its first return to this plane, similarly to the case of two-dimensional time-periodic flows. The plane used for this definition of the Poincaré map is often called a Poincaré plane. Furthermore, we again obtain Poincaré sections, by plotting several successive intersections of the trajectories of a few fluid particles with this plane. For a spatially periodic steady flow in an unbounded fluid region, such as the flows in static mixers, we usually use a series of planes whose interval is the same as the period of the flow as Poincaré planes. For static mixers, since all fluid particles generally move from the high-pressure side to the low-pressure side, it is common to select axial planes of interval $L$ as Poincaré planes. Therefore, the Poincaré map for spatially periodic steady flows in an unbounded region maps the initial position of a fluid particle on a Poincaré plane onto its location on a neighboring Poincaré plane at the time of its first arrival at this plane. On the basis of this Poincaré map, Poincaré sections for a spatially periodic flow are obtained.

We cannot always expect the existence of a Poincaré plane (or planes) that satisfies the condition described above. However, if this plane is obtained, we can easily distinguish chaotic and regular motions of fluid particles from Poincaré sections. The regular motion of a fluid particle on a curve that passes through the same point on Poincaré planes at its every $p$-th arrival corresponds to its periodic visit to each of a set of periodic 
points of a map $M$ of period $p$ in a Poincaré section. Moreover, the regular motion of a fluid particle on a torus or tubular region encircling this curve corresponds to its periodic visit to each of a set of $p$ closed lines around the above periodic points in a Poincaré section. The chaotic motion of a fluid particle is observed as an irregular motion of points in Poincaré sections.

It should be noted that the Poincaré map $M$ of motions of fluid particles by a threedimensional steady flow is usually not area-preserving. That is, although the volume of each fluid element is conserved, its cross-sectional area at its every intersection with a Poincaré plane varies because its perpendicular velocity usually depends on its crosssectional location. However, since the map $M^{p}$ is area-preserving in the neighborhood of its any periodic points of period $p$, it is expected that at least near the periodic points of map $M$ the motion of points in Poincaré sections is quite similar to that in Poincaré sections for two-dimensional time-periodic flows. That is, Eqs. (4)-(6) and the classification of periodic points into elliptic and hyperbolic ones described just below Fq. (6) hold also for the Poincaré map $M$ of three-dimensional steady flows if $z_{1}$ and $z_{2}$ are interpreted as two components of the position of a fluid particle on Poincaré planes and $t_{0}+p T$ in Eq. (4) is replaced by the time of the first return of the particle to these Poincaré planes. Therefore, as already explained, island regions are again expected to be observed around the elliptic periodic points of $M$ in Poincaré sections.

Studies of Lagrangian chaos by three-dimensional steady flows are again roughly classified into two groups, similarly to the case of two-dimensional time-periodic flows. In the first group, steady flow fields that are expressed in simple analytic forms but can yield Lagrangian chaos were chosen. These flows are convenient for the examination of dependences of this chaos on flow parameters. However, it is difficult to realize these flows in experiments, because supposed boundary conditions cannot be satisfied by any motion of rigid boundaries, or even because we have to give an unrealizable pressure distribution within a fluid. A typical example of such flows is the ABC flow (Dombre et al. 1986) defined in an unbounded region. The velocity field of this flow is expressed in a quite simple form by using sinusoidal functions, and is periodic in all the directions in Cartesian coordinates. Another example of such flows defined in a bounded region is the Stokes flow within a spherical droplet (Bajer and Moffatt, 1990). The last example of 
such flows is the flow in a twisted pipe. Jones et al. (1989) examined the cross-sectional chaotic motion of fluid particles as they move along a twisted pipe that is a periodic connection of several units composed of two semicircular pipe segments of circular crosssection connected with a twist. In this study, they used a discontinuous velocity field composed of the approximate velocity field of an analytic form in a pipe of constant curvature under a constant axial pressure gradient obtained by Dean $(1927,1928)$ in each segment.

In the studies of the second group, flows generated by a simple motion of rigid boundaries were used. Although these flows are expected to be realizable in experiments, their velocity fields are usually obtained only by numerical computations. Since velocity data of sufficiently high accuracy are required to obtain acceptable trajectories of fluid particles after long times, systematic numerical examinations of Lagrangian chaos for several values of flow parameters are not so easy if we use such numerical velocity fields. A typical example of such flows is the steady flow in a lid-driven cubic cavity (Ishii and Iwatsu, 1989, Iwatsu et al. 1989). This flow is produced by the motion of one boundary (lid) parallel to itself at a constant speed, and can yield Lagrangian chaos. The velocity fields used in studies of Lagrangian chaos and mixing in static mixers are also usually obtained only numerically because elements are included in the mixers.

One more subject to be discussed in this section is the dependence of Lagrangian chaos on the Reynolds number Re of flows that yield this chaos. In many studies on Lagrangian chaos, Stokes flows were used as velocity fields, and corresponding experiments were carried out at low Re by using the slow flow of highly viscous fluids such as glycerin. In two-dimensional time-periodic Stokes flows, since the inertial effect of fluid motion is neglected, symmetry with respect to time reversal is expected. That is, if we perform a 'return experiment' in which a flow boundary is periodically moved during a few periods and then is moved as its time reversal, the velocity field induced by this motion is expected to be symmetric with respect to time reversal. Therefore, the return of all the fluid particles advected by this flow to their initial locations is expected. However, since there are usually unavoidable disturbances, discrepancies between their initial and final locations may be observed. Chaiken et al. (1986) and Atobe et al. (1995) showed that the discrepancy for chaotic fluid particles is much larger than that for regular ones 
in the experimental and numerical studies of flows between eccentric rotating cylinders. Here by chaotic and regular fluid particles we mean that they are in chaotic and regular regions respectively in Poincare sections produced by the continuation of the periodic flow in the first half of the return experiment. Moreover, using the same flow, Aref and Jones (1989) numerically showed that when the effect of diffusion is included by a Brownian motion of fluid particles, much larger separation of a group of fluid particles initially in a small region is observed in the return experiment for chaotic fluid particles, compared with the case of regular fluid particles. Dutta and Chevray (1995) also carried out the return experiment experimentally and numerically using the same flow. They showed that the inertial effect associated with the variations in angular velocities of the cylinders on the separation of fluid particles is strong for chaotic fluid particles even at low Re. Therefore, the chaotic property of motions of fluid particles strongly destroys the deterministic property of this motion in a short time even if the disturbance to the expected flow is quite small.

Next, when Reynolds number Re is increased from zero, although flow fields vary with $R e$, they are still steady or time-periodic laminar flows up to a critical value of $R e$. Therefore, it is interesting to examine the dependence of Lagrangian chaos on $R e$ for $R e$ less than this value. In the study of Lagrangian chaos by steady flows in a lid-driven cubic cavity, the increase in the fraction of chaotic regions with Re was observed (Ishii and Iwatsu, 1989, Iwatsu et al. 1989). However, in the study of Lagrangian chaos by a steady flow in a model of static mixers, Mizuno and Funakoshi (2005) showed that the dependence of this fraction on $R e$ is not monotonic. No systematic investigation of this dependence is performed up to now.

Several studies on Lagrangian chaos by two-dimensional time-periodic flows or threedimensional steady flows are described or cited in two books by Ottino (1989) and by Sturman et al. (2006), and also in review articles by Ottino (1990), by Aref (1990, 1991, 2002), and by Cartwright et al. (1999).

\section{§3. Chaotic mixing and estimation of mixing efficiency}

Studies of chaotic mixing are usually based on the assumption that flows to mix fluids are independent of the configuration of the fluids. Therefore, although the cases in 
which this assumption is not satisfied, such as the mixing of fluids of different densities or viscosities, are also important in applications, we use this assumption in the present paper.

In the examination of mixing of fluids $\mathrm{A}$ and $\mathrm{B}$, we have to estimate the extent of mixing, that is how well these fluids are mixed. A primitive but important quantity used for this estimation is the normalized standard deviation $\sigma$ of volume ratio $r_{A}$ in many small regions of size $\delta$ obtained by dividing the region occupied by these fluids. That is, if $r_{A i}$ is the value of $r_{A}$ in the $i$-th small region, $\sigma$ is defined as

$$
\sigma=\sqrt{\frac{1}{N \bar{r}_{A}\left(1-\bar{r}_{A}\right)} \sum_{i=1}^{N}\left(r_{A i}-\bar{r}_{A}\right)^{2}}
$$

where $N$ is the total number of the small regions. Here $\sigma^{2}$ was called the intensity of segregation in the seminal paper by Danckwerts (1952). It is easily found that $\sigma$ is zero if these fluids are completely mixed at the length scale of $\delta$ and that $\sigma$ is equal to one if these fluids are completely separated. As was mentioned in Section 1, $\sigma$ depends on the value of $\delta$ and always tends to one in the limit of $\delta \rightarrow 0$ because we neglect diffusive effects. We usually have to use not too large and not too small a value of $\delta$ determined from the time provided for mixing and expected value of a diffusion constant.

Another quantity suggested for the estimation of the extent of mixing is the scale of segregation defined by Danckwerts (1952). This quantity gives a measure of the averaged linear or volume scale of blobs of fluid A (or fluid B) produced by the mixing of these fluids. When fluids A and B are mixed only insufficiently, the intensity of segregation and the scale of segregation express the characteristic amplitude and scale of the spatial variation of volume ratio $r_{A}$, respectively.

The value of $\sigma$ after the motion of fluids by a two-dimensional time-periodic flow for a specified number of periods is approximately calculated from the final locations of fluid particles initially distributed over the region of fluid A. Figure 2 shows an example of the particles' locations after five periods of the motion of cylinders for parameters used in Fig. 1. Here 3600 fluid particles are initially distributed in the lower half of the region between the cylinders. If fluid particles of sufficiently large number are initially uniformly distributed over the region of fluid A, we can obtain the approximate value of 


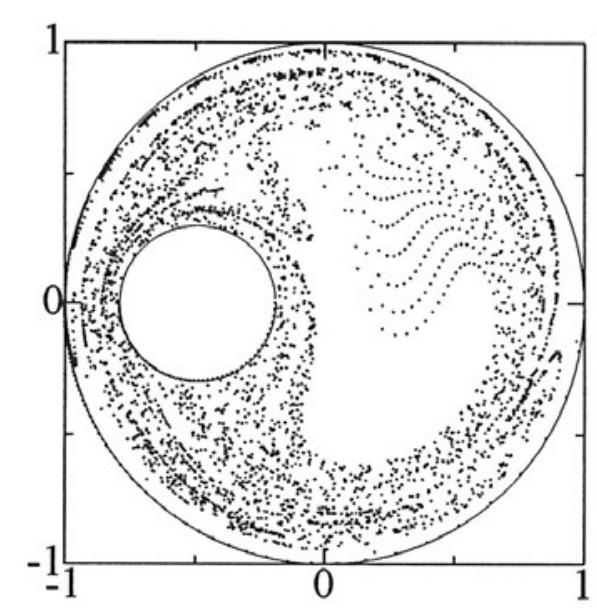

Figure 2: Locations of fluid particles after five periods of a time-periodic flow. $\alpha=$ $0.3, \varepsilon=0.7, T_{\text {out }}=T_{\text {in }}=1.0$. The definition of these parameters is given in Section 4 .

$r_{A i}$ for each small region from the number of the particles within it. Since Fig. 2 shows more homogeneous distribution of particles in the chaotic region of Fig. 1 compared with regular regions, efficient mixing within the chaotic region is suggested. Here it should be noted that if we use Poincaré planes to examine the mixing in their crosssectional directions by a three-dimensional steady flow, the dependence of normal fluid velocities on the cross-sectional positions on the planes must be taken into account in the computation of $\sigma$.

When we consider the mixing by a two-dimensional time-periodic flow, the value of $r_{A i}$ for each small region can be calculated more accurately from the information of curves expressing boundaries of fluids. However, in chaotic regions, the number of fluid particles required to express these curves with sufficient accuracy increases rapidly with the motion of fluids, because most of the line elements of these curves are stretched very fast (exponentially) reflecting the sensitive dependence on initial conditions of chaos. Therefore, it is not easy to obtain with high accuracy the configuration of boundary curves after the motion of fluids for many periods. Consequently, the computation of $\sigma$ based on boundary curves is not so efficient as expected first.

The length of boundary curves may be also suggested to estimate the extent of mixing. However, a large value of this length does not necessarily imply a well-mixed 
state, because the greatest fraction of the curves of large length is sometimes observed just within a few thin regions as many parallel lines with small spacing rather than extending over the whole region of the fluid. Therefore, this length is not a useful index of the extent of mixing.

One of the purposes of studies of mixing is to find a velocity field $\boldsymbol{u}(\boldsymbol{x}, t)$ that yields efficient mixing. For example, the $\boldsymbol{u}(\boldsymbol{x}, t)$ that reduces the value of $\sigma$ below a specified threshold value in the shortest time under the condition of a fixed amount of energy input per unit time required to generate it is the most preferable. That is, if a large fraction of the energy input to generate a flow is used for mixing rather than for a whole fluid motion such as a rigid rotation, the flow is efficient for mixing. However, it is difficult to define this fraction appropriately. Although the above definition of mixing efficiency seems appropriate, in the present paper we use the word of mixing efficiency without explicit discussion of the energy input to generate flows, under the assumption that the flows whose mixing efficiencies are to be compared are generated with a comparable amount of energy input per unit time.

Mixing efficiency of flows generally depends on the initial configurations of fluids to be mixed, because the value of $\sigma$ depends on them. Therefore, the selection of a flow of the highest mixing efficiency may be affected by initial configurations. Although the flow that has high mixing efficiency irrespective of initial configurations is the best if it exists, the most efficient flow under a special initial configuration is also important if this configuration is necessarily chosen from the structure of mixing systems. Another point of view in the consideration of mixing efficiency is the length of the time allowed for mixing. When mixing devices are used in factories or in laboratories of application areas, this length of time is sometimes not so large. For example, in some cases, the process of mixing should be finished within a specified time because this process is one of the successive processes of a production system. Moreover, the length of static mixers, which corresponds to the length of the time allowed for mixing, cannot be so large because of restrictions in space and in the total pressure difference. Therefore, in addition to the mixing efficiency of flows over a long time, that in a short time also should be examined. The dependence on initial configurations and the length of time allowed for mixing are important points of view in studies of mixing. In Section 4, several indices for the 
estimation of mixing efficiency in a short time will be introduced.

It is desirable if some information on mixing efficiency is obtained from velocity field $\boldsymbol{u}(\boldsymbol{x}, t)$ without solving Eq. (1). This information is obtained in a few exceptional cases. For example, efficient mixing is not expected for two-dimensional steady flows or twodimensional unsteady flows in which each fluid particle moves on a time-independent closed curve, because Lagrangian chaos is impossible in these cases. An example of the latter flows is the slow flow between two concentric cylinders that rotate alternately. For three-dimensional steady flows, a guideline in the search for Lagrangian chaos is the Beltrami property. That is, if the velocity $\boldsymbol{u}$ and vorticity $\boldsymbol{\omega}=\operatorname{rot} \boldsymbol{u}$ satisfy the relation $\boldsymbol{\omega} \times \boldsymbol{u} \neq \mathbf{0}$ everywhere in the fluid region, Lagrangian chaos is impossible, because any fluid particle is confined to a surface called the Lamb surface. However, if $\boldsymbol{\omega} \times \boldsymbol{u}=\mathbf{0}$ somewhere, chaos is possible. Moreover, the condition $\boldsymbol{\omega} \times \boldsymbol{u}=\mathbf{0}$ is satisfied everywhere when $\boldsymbol{u}$ has the Beltrami property of $\boldsymbol{\omega}=q \boldsymbol{u}$, where $q$ is any scalar function of $\boldsymbol{x}$. The $\mathrm{ABC}$ flow is a typical example of a flow with this property. However, unfortunately the Beltrami property is neither necessary nor sufficient for the existence of Lagrangian chaos. More detailed explanation of this problem was given by Aref et al. (1989). Another study of the relation between three-dimensional steady flow and Lagrangian chaos was performed by King et al. (2001) by using wavy Taylor-vortex flows. Guided by the fact that a flow with rotational symmetry and a flow satisfying $\operatorname{rot} \boldsymbol{\omega}=\mathbf{0}$ cannot yield Lagrangian chaos, they succeeded in predicting the dependence on Reynolds number of the effective axial diffusivity enhanced by Lagrangian chaos, on the basis of the product of a measure of the average deviation of $\boldsymbol{u}$ from this symmetry and a measure of the average deviation from the flow satisfying $\operatorname{rot} \boldsymbol{\omega}=\mathbf{0}$. Mizuno and Funakoshi (2005) also examined the relation between three-dimensional steady flows and Lagrangian chaos in a study of a model of static mixers.

One of the simplest data obtained from the numerical integration of Eq. (1) is a Poincaré section. As mentioned in Section 2, a region occupied by fluids for twodimensional flows or a Poincaré plane for three-dimensional flows is roughly divided into chaotic and regular regions. In chaotic regions, since the stretching and folding of boundary curves between fluids due to Lagrangian chaos are expected to produce a striped pattern with a small spacing, efficient mixing is expected. However, low mixing 
efficiency is predicted in regular regions, because the motion of fluid particles does not have a sensitive dependence on initial conditions. The most important property of the motion of fluid particles in Poincaré sections obtained from the theory of conservative dynamical systems is that particles starting from either of chaotic and regular regions never move into the other region. This property causes an inhomogeneously mixed state because the fluid in chaotic regions and that in regular regions are not mixed. Moreover, if there are two or more island regions in a Poincaré section, the fluid within one of these regions is never mixed with the fluid within any other island region. Therefore, the existence of regular regions is an obstacle to efficient mixing. Since a larger fraction of chaotic regions in the region occupied by fluids or in a Poincaré plane indicates more efficient mixing, this fraction is one of the useful indices of mixing efficiency, and is often used explicitly or implicitly in the studies of chaotic mixing.

The Poincaré section has a close relation to periodic points of the Poincaré map $M$, as explained in Section 2. Therefore, in studies of chaotic mixing, the locations and stability of these periodic points are sometimes calculated by solving Eq. (5) with the use of Newton's method and by calculating the eigenvalues of the Jacobian matrix $G$ defined by Eq. (6). The existence of elliptic periodic points of $M$ sometimes causes large island regions around them in a Poincaré section, and this should be avoided to attain efficient mixing. In contrast, most of hyperbolic periodic points of $M$ are observed in chaotic regions. Therefore, if the stability of such elliptic periodic points is lost by the change to hyperbolic type with the variation in a parameter included in $\boldsymbol{u}(\boldsymbol{x}, t)$, we usually expect that the island regions around them disappear through this bifurcation. Atobe and Funakoshi (1994) showed that chaotic regions become large when this bifurcation occurs with respect to one of the fixed points of $M$ in the study of the flows between eccentric rotating cylinders. Ling (1993) also carried out the bifurcation analysis of a few periodic points of $M$ to predict the ranges of a parameter within which the fraction of chaotic regions is quite high, in the study of three-dimensional flows in a model of static mixers. However, the loss of stability of an elliptic periodic point is sometimes associated with the appearance of other periodic points in pitchfork, symmetry-breaking, and period-doubling bifurcations, and also the appearance of new periodic points at a parameter value through a saddle-node bifurcation is often observed. Therefore, the 
selection of the periodic points whose stability and bifurcation are examined should be done quite carefully. Moreover, it is difficult to estimate the size of an island region around an elliptic periodic point only from the local information near this point such as the eigenvalues of $G$. It is often suggested that the periodic points of smaller period tend to have larger island regions around them (for example, Ottino and Shinbrot, 1999). This may be true to some extent in the sense that the island regions around periodic points of large period cannot be so large because their number is large. However, there seems to be no mathematical theory that is related to this suggestion. Moreover, this suggestion is often incorrect when we compare the island regions around periodic points of relatively small period such as 1, 2 and 3. Therefore, the stability and bifurcation analyses of periodic points of $M$ are not applicable to a wide range of mixing problems.

One more method for the estimation of mixing efficiency related to periodic points of $M$ is the examination of stable and unstable manifolds of hyperbolic periodic points of $M$ (Swanson and Ottino, 1990, Muzzio et al. 1992). The stable manifold $W^{s}$ or unstable manifold $W^{u}$ of such a periodic point $\boldsymbol{X}^{(p)}$ of period $p$ is one of the invariant sets of the map $M^{p}$. That is, the fluid particles starting from any locations on $W^{s}$ and $W^{u}$ move after every map $M^{p}$ only on these manifolds towards $\boldsymbol{X}^{(p)}$ as $t \rightarrow \infty$ and $t \rightarrow-\infty$, respectively. It is known that $W^{s}$ and $W^{u}$ are one-dimensional curves of infinite length that do not intersect with themselves except for the intersection of $W^{s}$ and $W^{u}$. Furthermore, it is also known that at the location of $\boldsymbol{X}^{(p)}, W^{s}\left(W^{u}\right)$ is tangent to the stable (unstable) direction, the direction of the eigenvector associated with the eigenvalue of Jacobian matrix $G$ of smaller (larger) absolute value. Hyperbolic periodic points are usually observed within a chaotic region if this region exists. Moreover, each of the stable and unstable manifolds of any hyperbolic periodic point is expected to have a complicated geometrical structure with an infinite number of folds, and to cover densely all the chaotic region without visiting regular regions. Therefore, the spread of one of these manifolds can be used as an index of mixing efficiency. That is, if this manifold extends over a larger part of the fluid region, higher mixing efficiency is expected because the fraction of chaotic regions is larger. $W^{s}\left(W^{u}\right)$ of $\boldsymbol{X}^{(p)}$ is usually obtained by integrating Eq. (1) in the backward (forward) direction from many initial points on a small line element oriented parallel to the stable (unstable) direction near $\boldsymbol{X}^{(p)}$, or more 
simply from many initial points on a small circle surrounding $\boldsymbol{X}^{(p)}$. Here, since this line element or circle is usually stretched exponentially with the increase in iterations of the mapping $M^{-p}$ or $M^{p}$, many points have to be added between initial neighboring points as it is stretched in order to calculate the manifold accurately. A large amount of computation is generally required to obtain a subset of $W^{s}$ or $W^{u}$ that is sufficient for the recognition of chaotic regions. Therefore, the computation of a subset of a stable or unstable manifold is not so useful to estimate the efficiency of mixing in a long time.

Efficient mixing is expected when most line fluid elements are stretched rapidly. Therefore, the stretching rate of these elements in a flow is often used as an index of mixing efficiency of this flow. The stretching rate $\lambda\left(\boldsymbol{x}, \theta, t_{e}\right)$ of an infinitesimal line element that is initially located at $\boldsymbol{x}$ and has initial orientation $\theta$ during the time $t_{e}$ is defined by

$$
\lambda\left(\boldsymbol{x}, \theta, t_{e}\right)=\frac{1}{t_{e}} \ln \left(\frac{d\left(\boldsymbol{x}, \theta, t_{e}\right)}{d(0)}\right),
$$

where $d\left(\boldsymbol{x}, \theta, t_{e}\right)$ and $d(0)$ are lengths of this line element at times $t_{e}$ and 0 , respectively. The maximum of the values $\lambda\left(\boldsymbol{x}, \theta, t_{e}\right)$ for all $\theta$ is expressed as $\lambda_{m}\left(\boldsymbol{x}, t_{e}\right)$, and is called a maximum stretching rate in the present paper. In the limit of $t_{e} \rightarrow \infty$, it is known that the values of $\lambda\left(\boldsymbol{x}, \theta, t_{e}\right)$ for almost all $\theta$ are the same as $\lambda_{m}\left(\boldsymbol{x}, t_{e}\right)$. Moreover, this long-time stretching rate of almost all the infinitesimal line elements, $\lambda_{m}(\boldsymbol{x}, \infty)=\lim _{t_{e} \rightarrow \infty} \lambda_{m}\left(\boldsymbol{x}, t_{e}\right)$, is the same as the largest Liapunov exponent commonly used in the study of chaos. Furthermore, $\lambda_{m}(\boldsymbol{x}, \infty)$ is independent of $\boldsymbol{x}$ if the ergodic property that any fluid particle moves all over the region of a fluid is satisfied. Therefore, if a chaotic region is observed in almost all of a Poincaré section, the value of $\lambda_{m}(\boldsymbol{x}, \infty)$ is almost independent of $\boldsymbol{x}$, because the ergodic property is usually expected within chaotic regions. In contrast to this, if both chaotic and regular regions are observed in a Poincaré section, the values of $\lambda_{m}(\boldsymbol{x}, \infty)$ for $\boldsymbol{x}$ within the chaotic and regular regions are a positive constant and zero, respectively, as expected from the sensitive dependence of chaos on initial conditions.

In comparison with the estimation of mixing efficiency based on Poincaré sections, the estimation based on the long-time stretching rate $\lambda_{m}(\boldsymbol{x}, \infty)$ is advantageous because we can estimate the efficiency quantitatively from the value of $\lambda_{m}(\boldsymbol{x}, \infty)$. For example, between globally chaotic flows, flows for which only chaotic regions are observed in 
Poincaré sections, the flow with the larger value of $\lambda_{m}(\boldsymbol{x}, \infty)$ is expected to have higher mixing efficiency because faster production of a striped pattern with a small spacing is expected in the whole region of a fluid. However, the estimation based on $\lambda_{m}(\boldsymbol{x}, \infty)$ has the disadvantage of the large amount of time required for the calculation of the field of $\lambda_{m}(\boldsymbol{x}, \infty)$. That is, we usually have to calculate $\lambda_{m}\left(\boldsymbol{x}, t_{e}\right)$ for sufficiently large $t_{e}$ at many points $\boldsymbol{x}$. In this computation at each $\boldsymbol{x}$, we have to integrate Eq. (1) for two neighboring fluid particles with frequent reductions of the distance between their trajectories by moving one of the particles so that the distance $d(\boldsymbol{x}, \theta, t)$ is small for all $0 \leq t \leq t_{e}$, or integrate six equations (for two-dimensional flows) or twelve equations (for three-dimensional flows) composed of Eq. (1) itself and the equations that govern the time evolutions of two or three components of the vector of an infinitesimal line element starting with two or three initial orientations. Therefore, although the maximum stretching rate $\lambda_{m}\left(\boldsymbol{x}, t_{e}\right)$ of line elements is one of the useful indices of mixing efficiency, as was shown in a few papers such as Swanson and Ottino (1990) and Muzzio et al. (1992), the computation of a field of this stretching rate over a long time is still not an easy job.

The indices of mixing efficiency described in this section and other indices not mentioned above were introduced in a book by Ottino (1989) and in a few review articles (Ottino, 1990, Aref, 1991, Wiggins and Ottino, 2004). There are also a few papers whose main purpose is the comparison of these indices (Swanson and Ottino, 1990, Finn et al. 2004).

\section{§4. Mixing efficiency in a short time}

The estimation of efficiency of mixing in a short time is also important, as explained in Section 1. Several indices of this efficiency will be described in this section.

\subsection{Classical indices of mixing efficiency}

First of all, the fraction of chaotic regions in Poincaré sections is still an important index of mixing efficiency even for mixing in a short time. For example, in the experi- 
ments on two-dimensional slow time-periodic flows between eccentric rotating cylinders by Chaiken et al. (1986), and on similar flows in a lid-driven cavity by Leong and Ottino (1989), it was shown that the spots or lines of dye initially located within chaotic regions are soon stretched and folded, whereas those located within regular regions are deformed only slightly. Chaiken et al. (1986) called the former and latter behaviors of such a dye tendril and whorl structures, respectively. These observations clearly show the decrease in mixing efficiency by the existence of regular regions. However, Poincaré sections cannot give any quantitative information on the mixing efficiency in chaotic regions. Therefore, if we would like to compare quantitatively the mixing efficiency in a short time of globally chaotic flows, other indices must be introduced.

One of the simplest methods to estimate the mixing efficiency in a short time is the examination of motions and deformations of small fluid blobs. If these blobs are strongly deformed to spread over a large region in a short time, high mixing efficiency is expected. For the quantitative estimation of this efficiency, the standard deviation $\sigma$ defined by Eq. (7) based on the configuration of these blobs is commonly used. The advantage and disadvantage of this method are the dependence on the initial locations of blobs. That is, if the initial locations of fluids to be mixed are fixed by the geometrical configuration of a mixing device, this dependence is advantageous in the sense that the direct estimation of mixing efficiency is possible. However, if their initial locations cannot be controlled, this dependence is disadvantageous because we have to examine the efficiency for several cases with different initial locations to perform reliable estimation. The estimation of mixing efficiency based on the time evolution of boundary curves has similar advantage and disadvantage. This method is more suitable for the mixing of fluids of comparable amounts, whereas the method based on the time evolution of small blobs is better for the mixing of a small amount of additives.

\subsection{Indices based on a transport matrix}

In a few studies on chaotic mixing by two-dimensional time-periodic flows, transport matrices were used to estimate its efficiency. In these studies, the region occupied by a fluid is divided into many small regions called cells. A transport matrix expresses 
what fraction of the fluid in a cell is transported to another cell by a given flow, and is also called a distribution matrix. Although the use of this matrix in the investigation of mixing was suggested early on (see, for example, Spencer and Wiley, 1951), studies attempting to extract information on mixing efficiency from this matrix started only recently (see, for example, Kruijt et al. 2001). An example of such studies (Funakoshi, 2001, Ichida and Funakoshi, 1998) of the slow flow between eccentric rotating cylinders will be shown below.

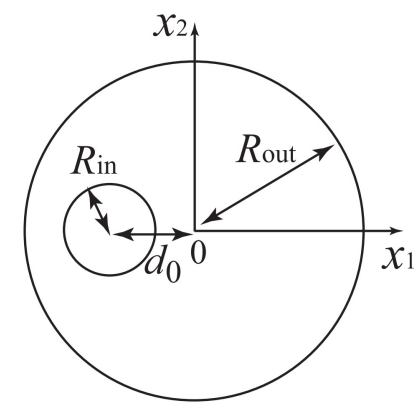

Figure 3: Geometrical configuration of the system of eccentric cylinders.

The geometrical configuration of the system of eccentric cylinders is shown in Fig. 3. The centers of outer and inner cylinders of radii $R_{\text {out }}$ and $R_{\text {in }}$ are located at $(0,0)$ and $\left(-d_{0}, 0\right)$ in the Cartesian coordinate system $\left(x_{1}, x_{2}\right)$, respectively. The two geometrical parameters of this system are the radius ratio $\alpha$ and eccentricity $\varepsilon$ defined by

$$
\alpha=\frac{R_{\text {in }}}{R_{\text {out }}}, \quad \varepsilon=\frac{d_{0}}{R_{\text {out }}-R_{\text {in }}} .
$$

Both $\alpha$ and $\varepsilon$ range from 0 to 1 . We consider the Stokes flow of an incompressible fluid between these cylinders caused by their alternate and periodic slow rotations with period $T$. Therefore, the parameters that characterize the rotations are the angles of rotation of outer and inner cylinders in one period, $T_{\text {out }}$ and $T_{\text {in }}$, respectively. In each period, the outer cylinder alone first rotates by $\pi T_{\text {out }}$ radians, and then the inner cylinder alone rotates by $2 \pi T_{\text {in }}$ radians, and finally the outer cylinder alone again rotates by $\pi T_{\text {out }}$ radians. Here the direction of these rotations is anticlockwise (clockwise) if $T_{\text {out }}$ or $T_{\text {in }}$ is positive (negative). The velocity field $\boldsymbol{u}(\boldsymbol{x}, t)$ of this Stokes flow is expressed in an 
analytic form by using a bipolar coordinate system, as was shown in Jeffery (1922) and in Ballal and Rivlin (1977). By integrating Eq. (1) with this $\boldsymbol{u}(\boldsymbol{x}, t)$, the motion of fluid particles and the deformation of material sets are obtained numerically.

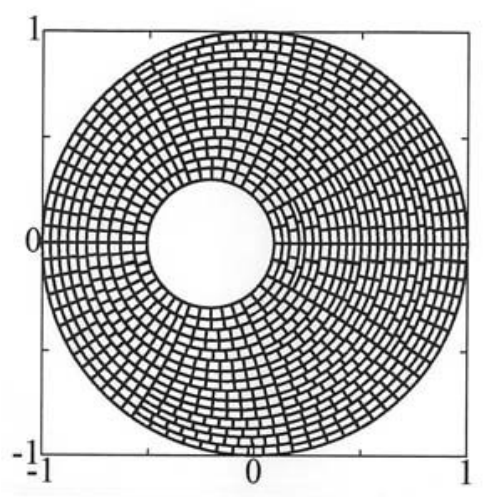

Figure 4: Example of the division of the region of a fluid into cells. $N=960 . \alpha=$ $0.3, \varepsilon=0.3$.

In order to examine the mixing efficiency of the flows described above by using a transport matrix $P$, we first divide the region occupied by a fluid into $N$ small cells of the same area $a$. An example of this is shown in Fig. 4. Here the region of a fluid is divided into 960 cells of approximately the same area.

The $(i, j)$ component $p_{i j}$ of the matrix $P$ expressing the transport of a fluid during a period $T$ is defined by $p_{i j}=a_{i j} / a$, where $a_{i j}$ is the area of the fluid that is in the $i$-th cell at $t=0$ and moves into the $j$-th cell at $t=T$ by $\boldsymbol{u}(\boldsymbol{x}, t)$. From this definition, $0 \leq p_{i j} \leq 1$ is satisfied for all $i$ and $j$, and the relations

$$
\sum_{j=1}^{N} p_{i j}=1 \quad(i=1,2, \ldots, N), \quad \sum_{i=1}^{N} p_{i j}=1 \quad(j=1,2, \ldots, N)
$$

are satisfied because the fluid is incompressible. The computation of the matrix $P$ is usually the most time-consuming task in the examination of mixing efficiency with a transport matrix. In the studies shown here, the locations of 2500 fluid particles initially distributed uniformly within the $i$-th cell after one period $T$ were calculated to obtain the values of $p_{i j}$ for each $i$. In other studies, more sophisticated methods were also 
used. For example, Kruijt et al. (2001) used a method called the adaptive boundary tracking, in which the motions of several fluid particles distributed on cell boundaries are calculated, and new particles are added on the initial cell boundaries and their motions are also calculated if the distance between neighboring evolved particles exceeds a threshold value that depends on the curvature of evolved boundary lines.

If we define $r_{A i}^{(n)}$ for non-negative integer $n$ as the area ratio of fluid $A$ in the $i$-th cell at $t=n T$ in the consideration of mixing of fluids $\mathrm{A}$ and B, the quantity $q_{i}^{(n)}=r_{A i}^{(n)} /\left(N \bar{r}_{A}\right)$ satisfies the relation

$$
\sum_{i=1}^{N} q_{i}^{(n)}=1
$$

for any $n$. Therefore, $\boldsymbol{q}^{(n)}=\left(q_{1}^{(n)}, q_{2}^{(n)}, \ldots, q_{N}^{(n)}\right)$ expresses the normalized distribution of fluid A at $t=n T$. Here we use the assumption that a series of transports each of which occurs in one period $T$ is a Markov process. That is, the relation

$$
\boldsymbol{q}^{(n)}=\boldsymbol{q}^{(0)} P^{n}
$$

is assumed for any positive integer $n$. This assumption corresponds to the complete mixing of fluids $\mathrm{A}$ and $\mathrm{B}$ in each cell with a specified ratio after every period $T$. Therefore, the use of Eq. (12) is associated with a diffusive effect. This effect is stronger for larger $a$ as was illustrated by Kruijt et al. (2001), and is not isotropic, unlike the case of usual diffusion. Therefore, we should be careful about this effect, especially when the mixing efficiency over a long time is examined. However, this effect seems negligible at least if we use Eq. (12) with sufficiently small $a$ to estimate the mixing efficiency in a short time.

If the use of Eq. (12) is allowed, mixing efficiency is estimated through a small amount of computations, because the estimation of the extent of mixing after a few periods by using an index such as $\sigma$ is carried out just by the multiplication of matrix $P$. Moreover, since all the information on the mixing ability of $\boldsymbol{u}(\boldsymbol{x}, t)$ is contained in $P$, we can estimate the mixing efficiency for several initial configurations of fluids to be mixed just by changing $\boldsymbol{q}^{(0)}$ in Eq. (12). Therefore, this method is useful especially when initial configurations cannot be fixed.

One of the indices related to the mixing efficiency in a short time is the distribution of eigenvalues of the matrix $P$. In order to examine these eigenvalues, we first assume 
that $P$ is irreducible and can be diagonalized as

$$
\Gamma=B^{-1} P B
$$

using a non-singular matrix $B$, where $\Gamma$ is a diagonal matrix whose $(i, i)$ component is $\lambda_{i} \quad(i=1,2, \ldots, N)$ satisfying the relation $\left|\lambda_{i}\right| \geq\left|\lambda_{j}\right|$ for any $i<j$. The irreducibility of $P$ is usually expected if we divide the region of a fluid into cells in a simple way. It is found from the Perron-Frobenius theorem that the eigenvalues $\lambda_{1}, \lambda_{2}, \ldots, \lambda_{N}$ satisfy the relation

$$
\lambda_{1}=1, \quad\left|\lambda_{i}\right| \leq 1 \quad(i=2,3, \ldots, N)
$$

Moreover, if the $(i, j)$ components of $B$ and $B^{-1}$ are expressed as $\alpha_{i j}$ and $\beta_{i j}$, respectively, we obtain

$$
P^{n}=\lambda_{1}^{n} H_{1}+\lambda_{2}^{n} H_{2}+\cdots+\lambda_{N}^{n} H_{N},
$$

from Eq. (13), where the $(i, j)$ component of the matrix $H_{l}$ is $\alpha_{i l} \beta_{l j}$ for $1 \leq i, j \leq N$ and $1 \leq l \leq N$. Here it is noted that all the components of $H_{1}$ are $N^{-1}$ and that the matrices $H_{l}$ are calculated from the eigenvectors of $P$. Therefore, if $\left|\lambda_{2}\right|<1$, we obtain

$$
\lim _{n \rightarrow \infty} P^{n}=H_{1} .
$$

Furthermore, using Eqs. (11), (12) and (16), the asymptotic state $\boldsymbol{q}^{(\infty)}$ of a normalized distribution vector is written as

$$
\boldsymbol{q}^{(\infty)}=\lim _{n \rightarrow \infty} \boldsymbol{q}^{(0)} P^{n}=\boldsymbol{q}^{(0)} H_{1}=\left(\frac{1}{N}, \frac{1}{N}, \ldots, \frac{1}{N}\right),
$$

independent of its initial value $\boldsymbol{q}^{(0)}$. Since this $\boldsymbol{q}^{(\infty)}$ corresponds to the ideally mixed state with $\sigma=0$, the speed of approach of $P^{n}$ to $H_{1}$ with increasing $n$ is important in the estimation of mixing efficiency in a short time. Therefore, from the consideration of Eq. (15), we conclude that the values of $\left|\lambda_{i}\right|$ for $i \geq 2$ can be used as an index of this mixing efficiency.

Figure 5 shows an example of the absolute values of $N$ eigenvalues of $P$ for five values of $\varepsilon$. As found from the Poincaré section for $\varepsilon=0.7$ shown in Fig. 1 and the Poincaré sections for $\varepsilon=0.5$ and 0.9 shown in Fig. 6, efficient mixing is expected for $\varepsilon=0.5$ because the fraction of chaotic regions is large, whereas low mixing efficiency is expected 


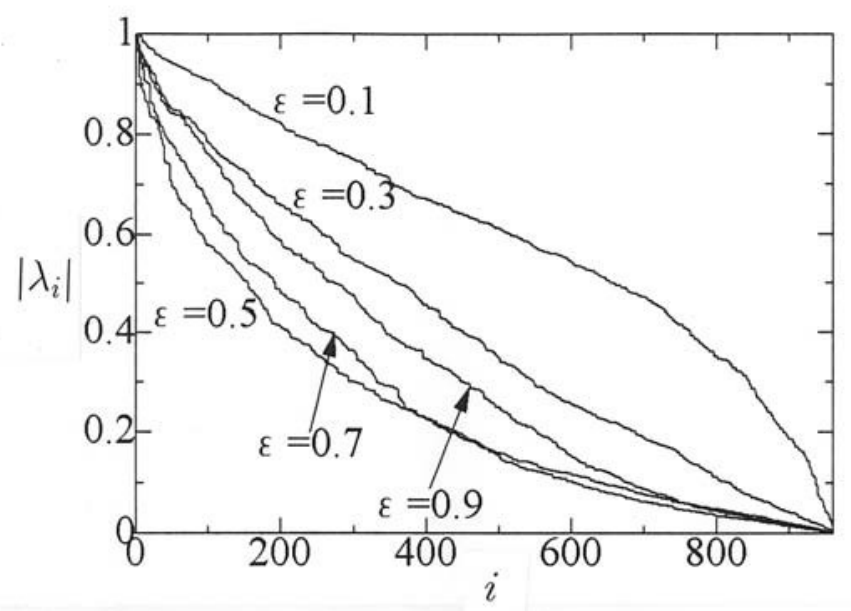

Figure 5: Absolute values of the eigenvalues of $P . \alpha=0.3, T_{\text {out }}=T_{\text {in }}=1.0$.
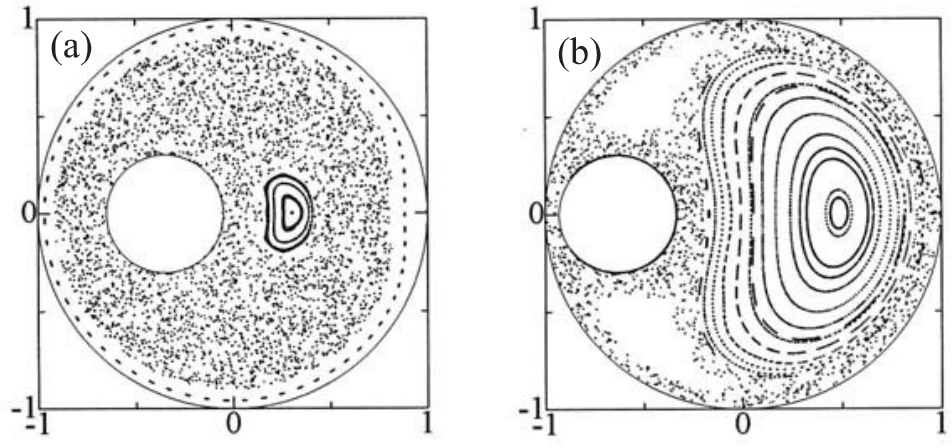

Figure 6: Examples of Poincaré sections. $\alpha=0.3, T_{\text {out }}=T_{\text {in }}=1.0$. (a) $\varepsilon=0.5$, (b) $\varepsilon=0.9$. 
if $\varepsilon$ is close to 0 or 1 because large regular regions are observed. The dependence of the distribution of eigenvalues on $\varepsilon$ in Fig. 5 is consistent with this result obtained from Poincaré sections. That is, the values of $\left|\lambda_{i}\right|$ for relatively small $i$ are the smallest for $\varepsilon=0.5$, and are large for $\varepsilon=0.1,0.3$ and 0.9 .

Although the direct estimation of mixing efficiency in a short time based on the values of $\left|\lambda_{i}\right|$ was not performed in this study, there are possibilities of extracting information on this mixing efficiency from $P$. For example, if $\left|\lambda_{3}\right|<\left|\lambda_{2}\right|<1$ is satisfied, matrix $H_{2}$ may give information on the pattern of inhomogeneity of fluids observed after the mixing in a short time. Anderson et al. (2002) also examined the mixing efficiency of the flow between eccentric rotating cylinders using the eigenvalues and eigenmodes of $P$. They discussed the relation between the eigenmodes associated with the eigenvalues whose absolute values are close to 1 and the configuration of chaotic and regular regions in the corresponding Poincaré sections.

It is also possible to characterize the contribution of each cell to mixing on the basis of $P$. That is, by analogy with entropy in information theory,

$$
L_{i}=-\frac{1}{\log N} \sum_{j=1}^{N} p_{i j} \log p_{i j} \quad(i=1,2, \ldots, N)
$$

is introduced as an index. If a fluid starting from the $i$-th cell is convected so that it is uniformly distributed over all cells after a period $T$, that is if $p_{i j}=N^{-1}$ for all $j$, then $L_{i}$ takes the largest value of 1 . However, if this fluid is convected to only one of the cells, $L_{i}$ takes the smallest value of 0 . Therefore, the cells of large $L_{i}$ are expected to play an important role in mixing because a small fluid blob visiting these cells is distributed over a large part of the region of a fluid after a period $T$. Figure 7 shows an example of the first $N / 5$ cells in descending order of $L_{i}$. As found from the corresponding Poincaré section of Fig. 1, almost all of these cells are within chaotic regions. Therefore, the large $L_{i}$ of these cells reflects the strong stretching of fluid elements in chaotic regions. Since high mixing efficiency in a short time is expected if $L_{i}$ is large for most cells, the spread of cells of large $L_{i}$ and the average of $L_{i}$ over all cells,

$$
\bar{L}=-\frac{1}{N \log N} \sum_{i=1}^{N} \sum_{j=1}^{N} p_{i j} \log p_{i j}
$$


are possible indices of this mixing efficiency.

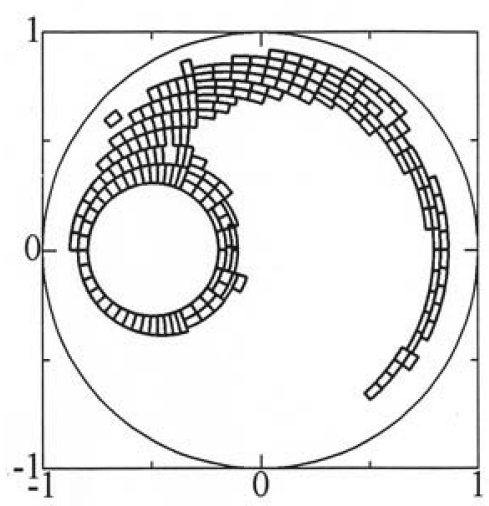

Figure 7: $N / 5$ cells with large $L_{i} . N=960 . \alpha=0.3, \varepsilon=0.7, T_{\text {out }}=T_{\text {in }}=1.0$.

Entropy in information theory can be used also for the estimation of the extent of mixing. That is, if we know the values of $q_{i}^{(n)}$,

$$
Q^{(n)}=\frac{-\log N-\sum_{i=1}^{N} q_{i}^{(n)} \log q_{i}^{(n)}}{\log \bar{r}_{A}}
$$

is introduced as another index of this extent at $t=n T$. In the completely mixed state of $q_{i}^{(n)}=1 / N$ for all $i, Q^{(n)}$ takes the smallest value of 0 . However, if fluids A and B are separated completely in the sense that $q_{i}^{(n)}=1 /\left(\bar{r}_{A} N\right)$ for $i=1, \ldots, \bar{r}_{A} N$ and $q_{i}^{(n)}=0$ for $i=\bar{r}_{A} N+1, \ldots, N$, then $Q^{(n)}$ takes the largest value of 1 , where $\bar{r}_{A} N$ is assumed to be an integer.

If the region occupied by a fluid could be divided into small cells so that some of the cells are within a chaotic region and the remaining cells are within a regular region, then the $(i, j)$ components $p_{i j}$ of transport matrix $P$ are zero if the $i$-th and $j$-th cells are in different regions, which means that $P$ is reducible. However, since the boundaries between chaotic and regular regions have a fractal structure as mentioned in Section 2, such a division is impossible, and the assumed irreducibility of $P$ is reasonable even when chaotic and regular regions coexist as in the case of Fig. 1. However, it is desirable if we can approximately detect these regions from this irreducible $P$ when both of them exist. This detection is possible to some extent by changing $P$ into a reducible one by 
replacing the values of components of $P$ that are smaller than a threshold value with zero. However, the selection of an appropriate threshold value is quite difficult, and may be even impossible in some cases. Therefore, we cannot expect the widespread availability of this method. If $P$ is reducible to two or more submatrices, the eigenvalues of $P$ are the union of the eigenvalues of these submatrices. Most of the eigenvalues of submatrices that correspond to the transport within regular regions are expected to be relatively large, whereas most of those corresponding to the transport within chaotic regions are expected to be relatively small. The property of eigenvalues of a near-reducible $P$, whose $(i, j)$ components are quite small if the $i$-th and $j$-th cells belong to different groups, seems an interesting subject and should be examined by using the perturbation theory for linear operators. However, this problem has not been examined systematically in the context of chaotic mixing.

Transport matrices were used in several studies on mixing efficiency. For example, Kruijt et al. (2001) estimated the mixing efficiency of two-dimensional time-periodic flows in a lid-driven cavity. Moreover, Galaktionov et al. (2001) examined the mixing efficiency of three-dimensional time-periodic flows in a cubic cavity generated by sliding opposite walls. Here this cavity was divided into many small cubic cells, and the surfaces of convected cells were expressed by a set of necessary number of triangles. A similar method was used also for the examination of mixing in a static mixer (Galaktionov et al. 2003). In these studies, the efficiency and accuracy of the method based on transport matrices in studies of mixing efficiency were shown.

\subsection{Indices related to important processes in mixing}

\subsubsection{General remarks on a few processes in mixing}

The estimations of mixing efficiency described in Sections 4.1 and 4.2 are based on the time evolution of material sets such as small blobs, boundary curves, and fluid particles, and are not directly related to the dynamical processes that are expected to play important roles in mixing. In contrast to them, in some studies, mixing efficiency was estimated by using the indices that characterize these processes, or the realization 
of high-efficiency mixing was attempted by taking account of these processes.

The most important dynamical process in mixing is the stretching of fluid elements. Therefore, it is ideal if we design a mixing device so that all fluid elements are always stretched strongly by a flow in it. Such a device was already realized as a split-andrecombine mixer in the study of static mixers at microscale, and its mixing efficiency was examined both numerically and experimentally (Hardt et al. 2006). The crosssectional motion of a fluid in this mixer after its axial motion by one period is expected to imitate the baker's transformation (see, for example, Wiggins and Ottino, 2004 about this transformation). The main processes in this mixer are stretching, cutting (splitting) and recombining (stacking) of the fluid. Hardt et al. (2006) showed that this mixer can attain high efficiency of mixing in a short time if its shape is carefully designed. However, the production of such a mixer usually costs much and is not easy, especially at microscale. Therefore, it is also important to estimate the efficiency of mixing devices of simpler geometrical configuration in which strong stretching of fluid elements is observed neither all over the region of a fluid nor all the time, such as typical static mixers used in many kinds of industries.

In estimating the mixing efficiency in a short time of such mixing devices, the maximum stretching rate $\lambda_{m}\left(\boldsymbol{x}, t_{e}\right)$ for small $t_{e}$, which is sometimes called a finite-time largest Liapunov exponent, is one of the useful indices. For example, through the experiments and numerical computations of the time-periodic flows between eccentric rotating cylinders, Swanson and Ottino (1990) showed that the configuration of small spots of dye initially located in a chaotic region after a few periods agrees well with the region composed of final locations of the fluid elements of large stretching rate. Here they used the value of $\lambda\left(\boldsymbol{x}, \theta, t_{e}\right)$ for small $t_{e}$ averaged over $\theta$ as the stretching rate instead of $\lambda_{m}\left(\boldsymbol{x}, t_{e}\right)$.

However, since stretching is not the only important process in mixing, we have to consider other processes too. One of the traditional strategies to achieve efficient mixing is strong stretching of fluid elements in relatively small regions and the successive repeated visits of all fluid elements to these regions after every interval. In this strategy, if fluid elements in a large part of the region of a fluid do not visit the high-stretching-rate regions, efficient mixing is not expected. Furthermore, in addition to the magnitude of the stretching rate in these regions, the orientation of line fluid elements when they return to 
these regions is also important. That is, since the stretching rate of a line element visiting a high-stretching-rate region depends on this orientation, its return with the orientation that gives the largest stretching rate is the most preferable. In the estimation of mixing efficiency of three-dimensional steady flows, the shear rates of these flows are usually examined to characterize stretching rates. However, the stretching rate of line fluid elements by a Poincaré map $M$ is also useful for the estimation of cross-sectional mixing efficiency of three-dimensional steady flows if such a map is defined well. Moreover, for two-dimensional time-periodic flows, it is more reasonable to examine the stretching rates by a Poincaré map $M$ rather than the time-dependent shear rates of these flows.

\subsubsection{Stable and unstable manifolds}

One of the candidates for a high-stretching-rate region for a two-dimensional timeperiodic flow is the neighborhood of a hyperbolic periodic point of a Poincaré map $M$. That is, the strong stretching of fluid elements is expected near the hyperbolic periodic points with unstable eigenvalue $\mu_{1}$ of large absolute value, as was suggested by Muzzio et al. (1992). The stable manifolds of these periodic points are interpreted as the skeleton of the locations of fluid particles that are promised to visit the neighborhoods of these periodic points. On the other hand, the unstable manifolds of these periodic points are interpreted as the skeleton of the locations of fluid particles that were strongly stretched in the neighborhoods of these periodic points. Therefore, the distribution of appropriately defined subsets of these manifolds can be used as an index of the efficiency of mixing in a short time. For example, in the studies on the time-periodic flow between eccentric rotating cylinders, Swanson and Ottino (1990) and Muzzio et al. (1992) showed that the configuration of small spots of dye initially located in a chaotic region after a few periods obtained in their experiments is predicted well by subsets of unstable manifolds of one or more hyperbolic fixed points with large $\left|\mu_{1}\right|$.

Another example of the study of the estimation of efficiency of mixing in a short time based on the distribution of subsets of stable and unstable manifolds will be shown below (Kawazoe et al. 2004, Kawazoe, 2004). In this study, the flow between eccentric rotating cylinders for $\alpha=0.3, \varepsilon=0.4, T_{\text {nut }}=1.0$ and $T_{\text {in }}=6.0$ was mainly used. The 


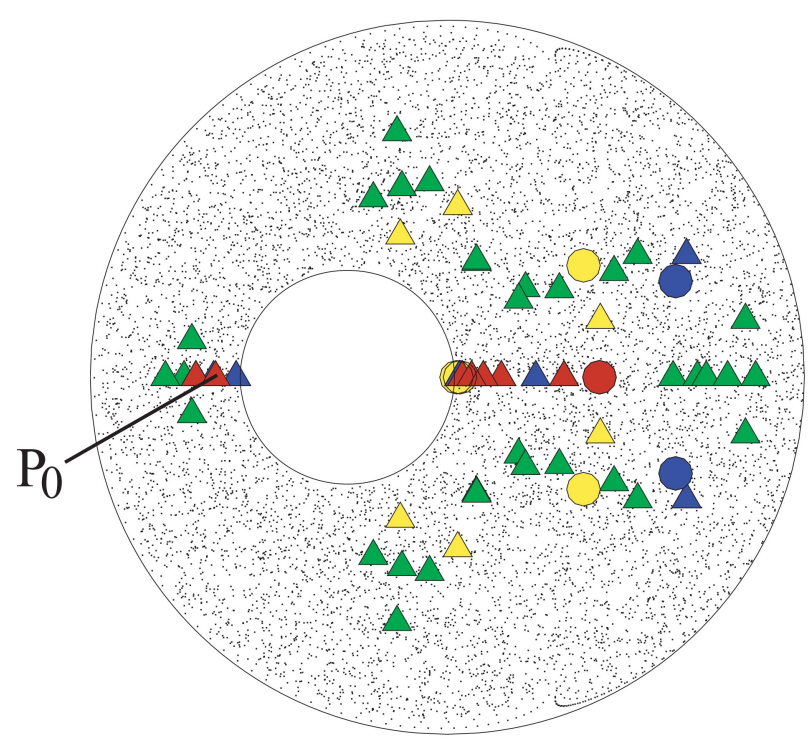

Figure 8: Poincaré section and periodic points of the Poincaré map $M$ for $\alpha=0.3, \varepsilon=$ $0.4, T_{\text {out }}=1.0$ and $T_{\text {in }}=6.0$. Periodic points are colored red for fixed points, blue for 2-periodic points, green for 3-period points, and yellow for 4-period points. Circles and triangles denote elliptic and hyperbolic periodic points, respectively.

Poincaré section and $p$-periodic points of the Poincaré map $M$ satisfying $p \leq 4$ yielded from this flow are shown in Fig. 8. We refer to periodic points of period $p$ as $p$-periodic points in the following part. Since chaotic regions are dominant in this figure, the flow we consider is globally chaotic. Most of the periodic points we found are hyperbolic. Since island regions around a few elliptic periodic points are quite small, they are not recognizable in the Poincaré section.

At hyperbolic $p$-periodic points, the stretching rate of a line element oriented to the unstable direction in $p$ periods is given by $\ln \left|\mu_{1}\right|$, where $\mu_{1}$ is the unstable eigenvalue of the Jacobian matrix $G$ of map $M^{p}$ at these points. The maximum stretching rate of line elements in $p$ periods at these points is given by $\ln \Lambda_{1}$. Here $\Lambda_{1}$ is the square root of the larger eigenvalue of the Cauchy-Green tensor $G^{\mathrm{t}} G$, where t denotes the transpose. Although $\Lambda_{1}$ is larger than $\left|\mu_{1}\right|$ if $G$ is asymmetric, $\left|\mu_{1}\right|$ is still an important measure to characterize the stretching rate at these points, because the asymptotic stretching rate per one period is given by $\frac{1}{p} \ln \left|\mu_{1}\right|$ rather than $\frac{1}{p} \ln \Lambda_{1}$. The existence of hyperbolic 
periodic points of large $\frac{1}{p} \ln \left|\mu_{1}\right|$ or $\frac{1}{p} \ln \Lambda_{1}$ is preferable for efficient mixing, because strong stretching is expected near these points. We usually find that most of the hyperbolic periodic points with large $\frac{1}{p} \ln \left|\mu_{1}\right|$ or $\frac{1}{p} \ln \Lambda_{1}$ have small period such as one, two and three. In the case of Fig. 8, the fixed point expressed by $\mathrm{P}_{0}$ gives the largest values of $\frac{1}{p} \ln \left|\mu_{1}\right|$ and $\frac{1}{p} \ln \Lambda_{1}$ among hyperbolic periodic points of $M$. Therefore, we call $\mathrm{P}_{0}$, for which $\left|\mu_{1}\right|=45.3$, a strongest-stretching fixed point.

Each hyperbolic $p$-periodic point $\boldsymbol{X}^{(p)}$ has stable manifold $W^{s}$ and unstable manifold $W^{u}$, as already explained in Section 3. As illustrated in Fig. 9, a circular fluid element whose center is initially on $W^{s}$ is usually stretched along $W^{u}$ and compressed in the direction of $W^{s}$. Moreover, strong stretching of this fluid element is expected if $\frac{1}{p} \ln \left|\mu_{1}\right|$ associated with $\boldsymbol{X}^{(p)}$ is large. Therefore, the fluid near a subset of $W^{s}$ of such an $\boldsymbol{X}^{(p)}$ is expected to be mixed well in a short time if fluid particles starting from this subset arrive at the neighborhood of $\boldsymbol{X}^{(p)}$ in a few periods.

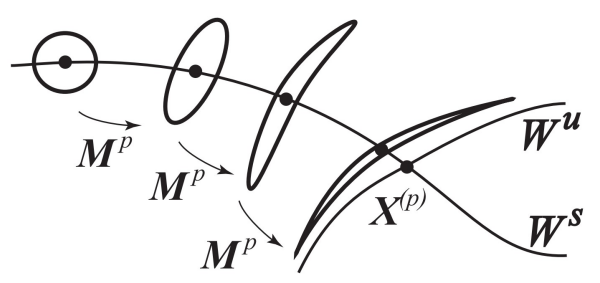

Figure 9: Motion and deformation of a fluid element that is initially on the $W^{s}$ of a hyperbolic $p$-periodic point $\boldsymbol{X}^{(p)}$ by the map $M^{p}$.

In order to obtain an approximation of such a subset of $W^{s}$, the mapping $M^{-k}$ of a circle of small radius $\delta$ around $\boldsymbol{X}^{(p)}$ is carried out, where $k$ is assumed to be a multiple of $p$. The resulting set, which usually looks like a curve with two ends, is called $S_{k, \delta}$. An approximation of a subset of $W^{u}$ is obtained in a similar way by using map $M^{k}$ instead of $M^{-k}$, and is called $U_{k, \delta}$. Figure 10 shows examples of $S_{4, \delta}$ for the strongest-stretching fixed point $\mathrm{P}_{0}$ expressed by a small square. We find that the length of $S_{k, \delta}$ increases quite rapidly with $\delta$, and that even for relatively large $\delta, S_{k, \delta}$ passes through only narrow regions frequently without visiting the remaining region rather than visiting most of the chaotic region. Here it should be noted that $U_{k, \delta}$ is the mirror image of $S_{k, \delta}$ with respect to the axis of symmetry $\left(x_{2}=0\right)$, because the inverse of map $M$ corresponds to the 
time-reversal rotations of cylinders.

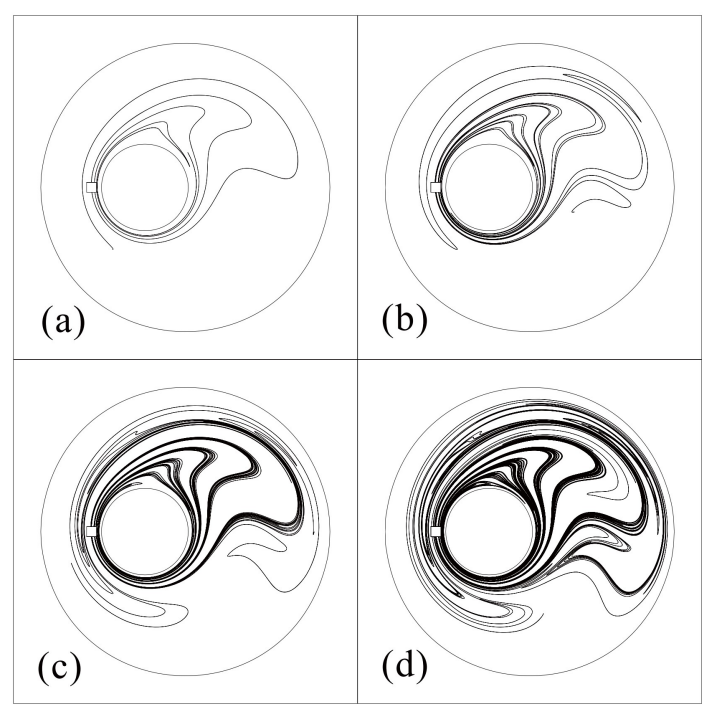

Figure 10: Dependence of $S_{k, \delta}$ on $\delta . \alpha=0.3, \varepsilon=0.4, T_{\text {out }}=1.0, T_{\text {in }}=6.0$ and $k=4$. (a) $\delta=10^{-6} R_{\text {out }}$, (b) $\delta=10^{-5} R_{\text {out }}$, (c) $\delta=10^{-4} R_{\text {out }}$, (d) $\delta=10^{-3} R_{\text {out }}$.

To compare the stable manifolds of different periodic points, the $S_{k, \delta}$ for three fixed points including $P_{0}$ is shown in Fig. 11. The values of $\left|\mu_{1}\right|$ of fixed points in (a), (b) and (c) of this figure are 45.3, 17.3 and 9.8, respectively. For fixed values of $k$ and $\delta$, the $S_{k, \delta}$ for the fixed points with larger $\left|\mu_{1}\right|$ is distributed over a larger region. Moreover, for sufficiently large $k$ and $\delta$, the configurations of $S_{k, \delta}$ for these fixed points are similar. Therefore, we examine only the $S_{k, \delta}$ for $\mathrm{P}_{0}$ in the following part.

To estimate the density distribution of $S_{k, \delta}$ quantitatively, we first divide the region of the fluid into $N_{c}=28000$ small cells of equal area. We then count the number of times, $f_{i}$, the curve of $S_{k, \delta}$ passes through the $i$-th cell. The density $F_{i}$ of $S_{k, \delta}$ at the $i$-th cell is defined by

$$
F_{i}=\frac{f_{i}}{\sum_{j=1}^{N_{c}} f_{j}} .
$$

Figure 12 shows examples of the density distribution of $S_{4, \delta}$ for a few values of $\delta$. Since the distributions for relatively large $\delta$ are similar to each other, it is suggested that there exists an asymptotic distribution of $S_{k, \delta}$ as $\delta$ is increased for fixed $k$ or as $k$ is increased 
(a)
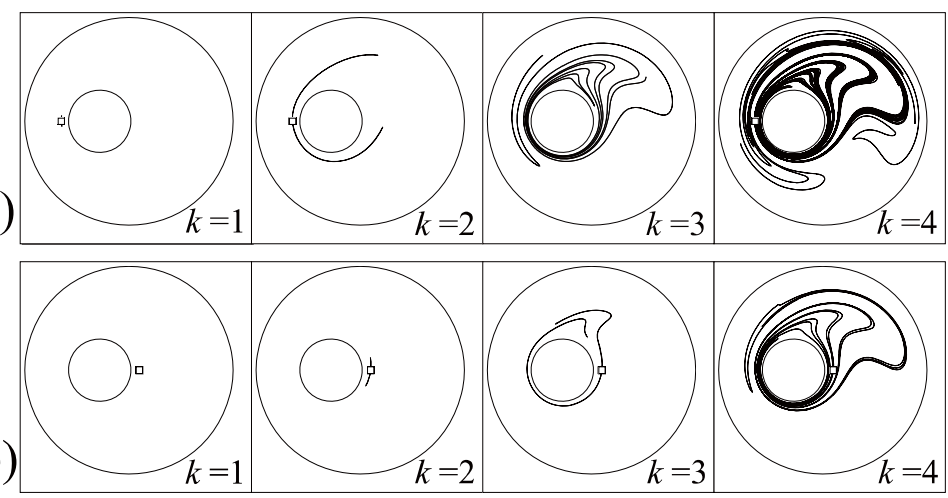

(b)

$$
k=1 \quad k=2 \quad k=3 \quad k=4
$$

(c)

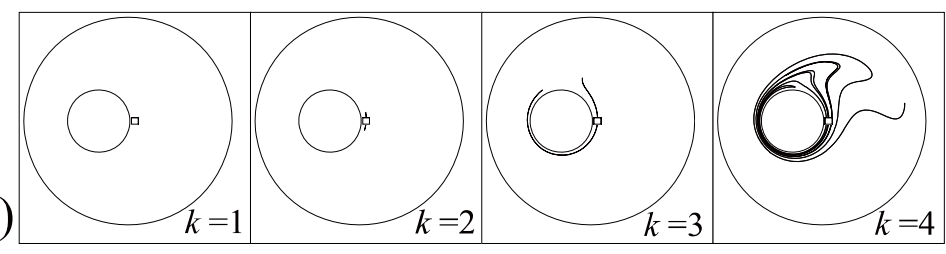

Figure 11: $S_{k, \delta}$ for three fixed points denoted by squares. $\alpha=0.3, \varepsilon=0.4, T_{\text {out }}=$ 1.0, $T_{\text {in }}=6.0$ and $\delta=10^{-4} R_{\text {out }}$. The fixed point in (a) is $\mathrm{P}_{0}$.

for fixed $\delta$. This distribution may characterize the configuration of $W^{s}$.

To examine the validity of the distribution of $S_{k, \delta}$ as an index of the efficiency of mixing in a short time, the time evolutions of small blobs located at the regions of large and small $F_{i}$ are compared in Fig. 13. From this figure, we find that the blob starting from the region of large $F_{i}$ is stretched strongly and is distributed over a large region, whereas the other blob is stretched only weakly. Moreover, the configuration of the strongly stretched blob shown in Fig. 13(b) is similar to the unstable manifold of $\mathrm{P}_{0}$. This result is consistent with the expected behavior of fluid elements shown in Fig. 9. Therefore, it is suggested that the distribution of $S_{k, \delta}$ (or $U_{k, \delta}$ ) for appropriately chosen $k$ and $\delta$ is one of the useful indices of the efficiency of mixing in a short time. If $S_{k, \delta}$ (or $U_{k, \delta}$ ) is distributed over a large part of the region of a fluid without concentrating in narrow regions, efficient mixing in a short time is expected.

In order to estimate quantitatively the suggested strong stretching effect of a flow near hyperbolic periodic points of $M$, the maximum stretching rate $\lambda_{m}\left(\boldsymbol{x}, t_{e}\right)$ at several points $\boldsymbol{x}$ is also calculated for $t_{e}=n T$, where $n$ is a positive integer. That is, we first calculate $\Lambda_{1}$ as the square root of the larger eigenvalue of $G^{\mathrm{t}} G$, where $G$ is the Jacobian 


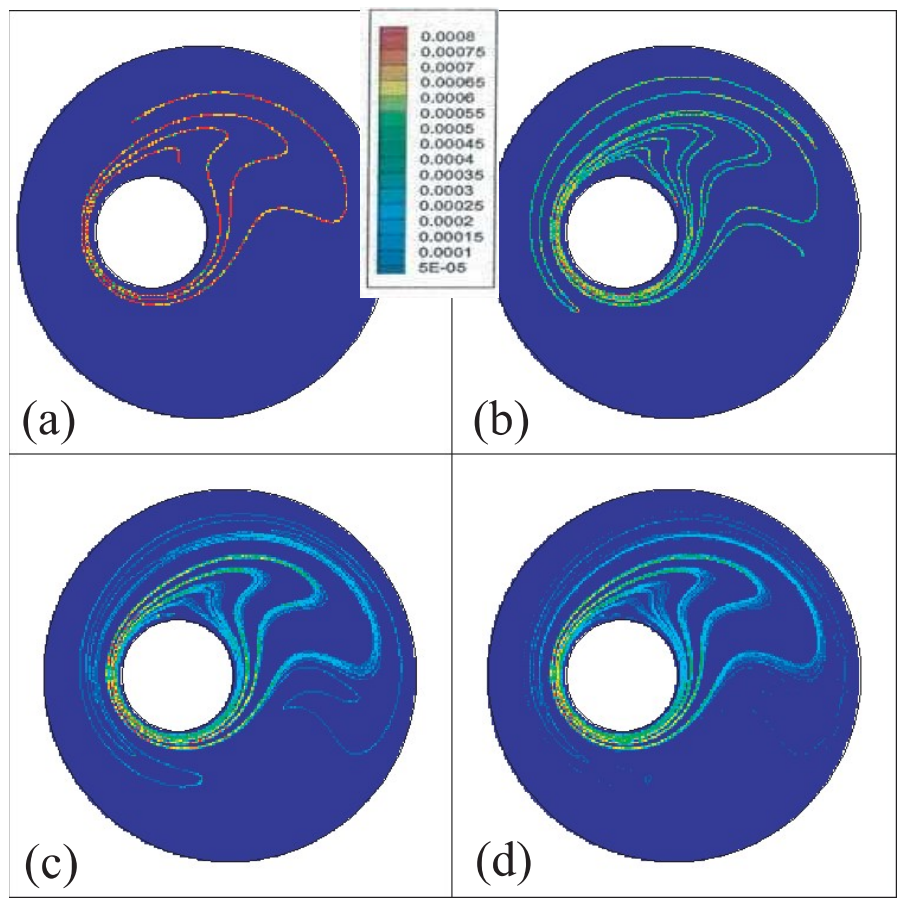

Figure 12: Density distribution of $S_{k, \delta}$ for $\mathrm{P}_{0} . \alpha=0.3, \varepsilon=0.4, T_{\text {out }}=1.0, T_{\text {in }}=6.0$ and $k=4$. (a) $\delta=5 \times 10^{-7} R_{\text {out }}$, (b) $\delta=5 \times 10^{-6} R_{\text {out }}$, (c) $\delta=5 \times 10^{-5} R_{\text {out }}$, (d) $\delta=5 \times 10^{-4} R_{\text {out }}$. 


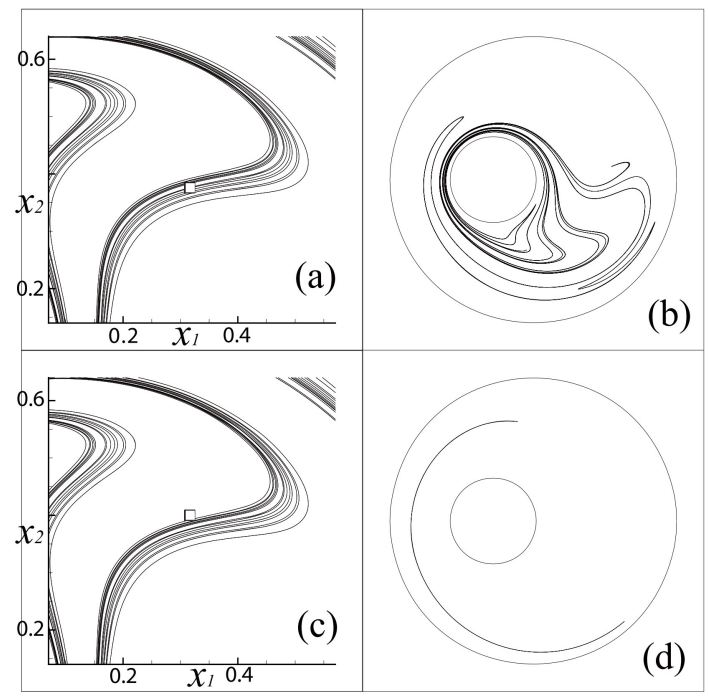

Figure 13: Time evolutions of two small blobs. $\alpha=0.3, \varepsilon=0.4, T_{\text {out }}=1.0$ and $T_{\text {in }}=6.0$. In (a) and (c), a part of the $S_{k, \delta}$ for $\mathrm{P}_{0}$ for $k=4$ and $\delta=10^{-4} R_{\text {out }}$ is shown. Small squares denote the initial positions of circular blobs of radius $10^{-3} R_{\text {out }}$. These blobs in (a) and (c) evolve into the curves in (b) and (d) after 4 periods, respectively.

matrix of map $M^{n}$ that corresponds to the motion of a fluid particle starting from $\boldsymbol{x}$ in time $n T$. We then obtain $\lambda_{m}(\boldsymbol{x}, n T)$ from

$$
\lambda_{m}(\boldsymbol{x}, n T)=\frac{1}{n} \ln \Lambda_{1} .
$$

The maximum stretching rate in $n$ periods at each cell used in the computation of $F_{i}$ is defined as the averaged value of $\lambda_{m}\left(\boldsymbol{x}_{j}, n T\right)$ for several points $\boldsymbol{x}_{j}$ within this cell, and is referred to as $\lambda_{i}(n T)$ for the $i$-th cell.

Figure 14 shows examples of the distribution of $\lambda_{i}(n T)$ for $n=1,2,3$ and 4 . Although the difference between the largest and smallest values of $\lambda_{i}(n T)$ tends to decrease as $n$ increases, the locations of the cells with relatively large $\lambda_{i}(n T)$ for different values of $n$ are similar, especially between the locations for $n=3$ and 4 . Moreover, the locations of the cells with large $\lambda_{i}(n T)$ in Fig. 14 look similar to those with large density of $S_{k, \delta}$ in Fig. 12 to some extent. To examine the correlation between the cells with large $\lambda_{i}(n T)$ and the ones with large $F_{i}$, the matching rate of such cells is computed for several values of $\delta$ and $n$. That is, we calculate the proportion of the cells that are within the first 


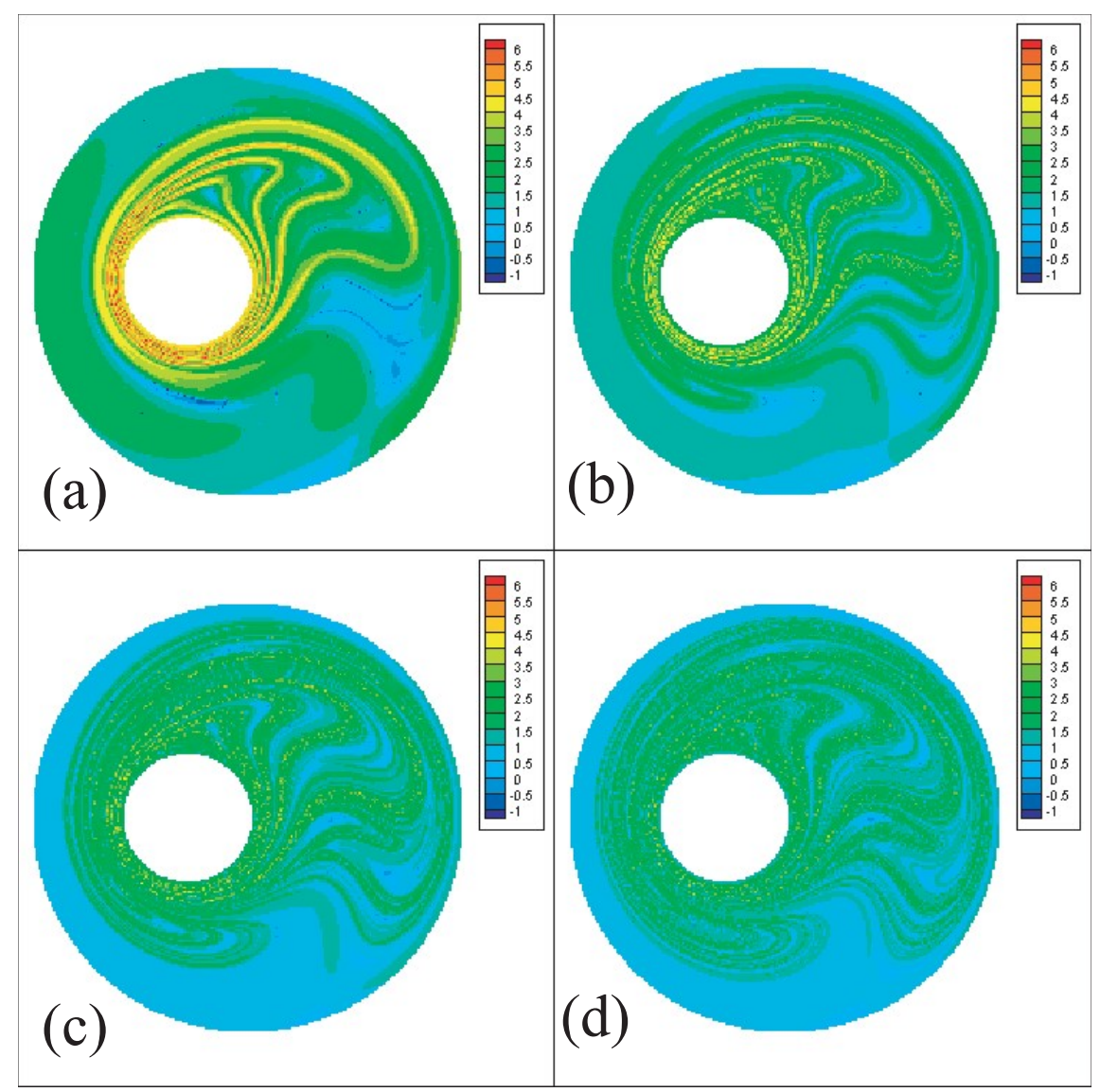

Figure 14: Distribution of $\lambda_{i}(n T) . \alpha=0.3, \varepsilon=0.4, T_{\text {out }}=1.0$ and $T_{\text {in }}=6.0$. (a) $n=1$, (b) $n=2$, (c) $n=3$, (d) $n=4$. 


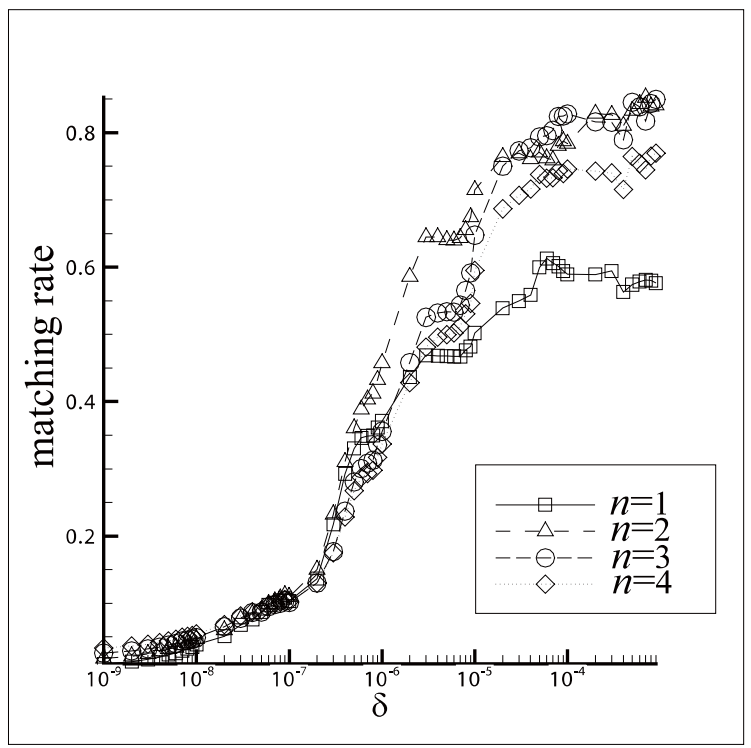

Figure 15: Matching rate of the cells with large $F_{i}$ of $S_{4, \delta}$ and the cells with large $\lambda_{i}(n T)$.

$10 \%$ of the cells in descending order of $F_{i}$ and also are within the first $10 \%$ of the cells in descending order of $\lambda_{i}(n T)$, to $10 \%$ of all cells. Figure 15 shows the matching rate between the distribution of density $F_{i}$ of $S_{4, \delta}$ illustrated in Fig. 12 and the distribution of $\lambda_{i}(n T)$ shown in Fig. 14. From Fig. 15, we find that this rate is high if $\delta$ is not too small and $n$ is two or more. Therefore, we can conclude that if the values of $k$ and $\delta$ are appropriately chosen, most of the fluid elements near $S_{k, \delta}$ are strongly stretched and distributed over a large region in a short time. An almost exact correspondence between the region of large stretching rate and the stable manifolds of hyperbolic fixed points with large $\left|\mu_{1}\right|$ was also shown by Muzzio et al. (1992).

The stable and unstable manifolds of hyperbolic fixed points of the map $M$ were also used to estimate the rates of transport and mixing of fluids by two-dimensional flows expressed as a time-periodic modulation of a steady velocity field. Here it is assumed that the steady velocity field yields streamlines that separate the region of a fluid and connect stagnation points. Such a separating streamline is interpreted as the stable manifold of an equilibrium point and also the unstable manifold of another equilibrium point. When this steady velocity field is slightly modulated periodically, fixed points of 
the Poincaré map $M$ exist near these equilibrium points. Moreover, the stable manifold of one of these fixed points and the unstable manifold of another fixed point no longer coincide and intersect only at infinite number of discrete points. This behavior is called the heteroclinic tangle of stable and unstable manifolds. Rom-Kedar et al. (1990) showed that the regions called lobes are important in estimating the transport rate of a fluid beyond the above separating streamlines. Here each lobe is delimited by a part of the stable and unstable manifolds connecting two neighboring intersection points. On the basis of the dynamics of lobes, several studies were performed on the rates of transport and mixing of fluids by this kind of flow (see for example, Wiggins 1992, Beigie et al. 1994, Wilson et al. 2006).

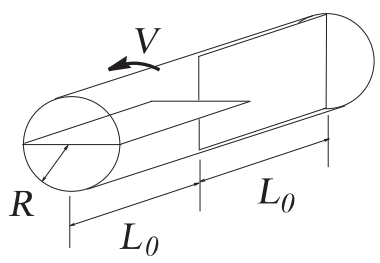

Figure 16: Schematic view of one period of the partitioned-pipe mixer.

\subsubsection{Lines of separation in a static mixer}

As a simple model of static mixers, the partitioned-pipe mixer (PPM) was first introduced by Khakhar et al. (1987). As shown in Fig. 16, the elements within the PPM are a series of rectangular plates of length $L_{0}$ that are placed orthogonally to each other. In this system, a cross-sectional flow is caused by the rotation of an outer cylinder of radius $R$ relative to the elements at constant speed $V$ instead of the torsion of elements, and an axial flow is generated by the imposed pressure gradient in the axial direction. Khakhar et al. (1987) examined numerically the mixing in the PPM as a case study of chaotic mixing in deterministic flows, using the approximate Stokes flow velocity field that is expressed in an analytical form but is discontinuous on the cross-sections that include the leading and trailing edges of plates. The mixing in the PPM was also studied 
experimentally by Kusch and Ottino (1992). By examining the spatial development of injected dyed fluids, they observed both the regions of chaotic motion and the tubular regions extending in the axial direction that correspond to regular regions explained in Section 2. Using an improved velocity field that is still discontinuous on the same crosssections, Meleshko et al. (1999) also performed numerical studies on the mixing in the PPM and showed that their results agree well with the results in experiments by Kusch and Ottino (1992). Mizuno and Funakoshi (2002) used the same improved velocity field in the study of mixing in a generalized model of the PPM. Moreover, numerically obtained spatially-periodic continuous velocity fields were used by Mizuno and Funakoshi (2004, 2005) in a study of mixing in this generalized model.

In typical static mixers including the PPM, the fluid in a mixer is separated (or split) at the leading edge of each element. The cross-sectional motions of the separated fluids on the opposite sides of the element are toward the opposite directions along its surface, which results in the discontinuity of Poincaré map $M$. Moreover, the fluid near elements is often stretched strongly in the cross-sectional directions because of the large strain rate of the cross-sectional velocity field. Therefore, the separation of fluid at the leading edges of elements is expected to be one of the important processes whose characteristics are used to estimate the mixing efficiency in such static mixers.

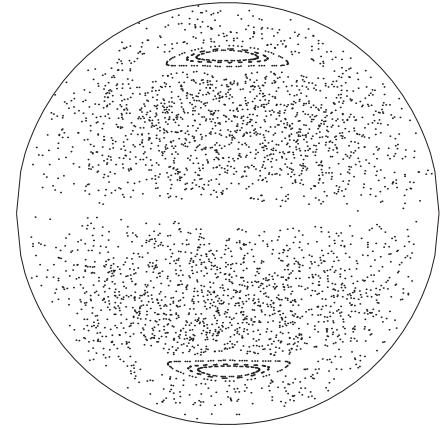

(a)

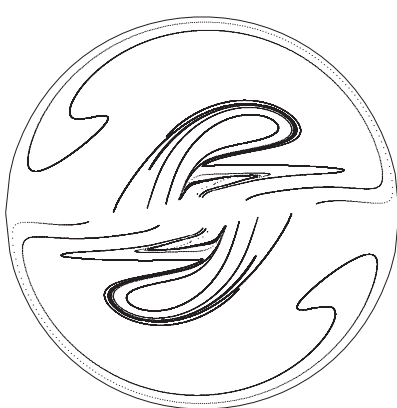

(b)

Figure 17: (a) Poincaré section, (b) lines of separation.

On the basis of this expectation, Mizuno and Funakoshi (2002, 2004, 2005) suggested that the distribution of a set, called lines of separation, is one of the useful indices to estimate the efficiency of mixing in the PPM in a short time. Lines of separation are 
defined as the set of locations on a given cross-section of fluid particles that are advected to one of the leading edges of plates within a specified number of periods. Figure 17 shows examples of the Poincaré section and lines of separation in the PPM yielded by a numerically obtained velocity field for certain values of non-dimensional parameters composed of $L_{0}, R, V$, pressure gradient and viscosity (Mizuno and Funakoshi, 2004). From the Poincaré section shown in Fig. 17(a), defined at cross-sections $Z_{m}$ that correspond to the middle of horizontal plates, we find that the flow is almost globally chaotic, since island regions are small. Figure 17(b) shows the lines of separation on the same cross-section $Z_{m}$ obtained from two leading edges within one period from $Z_{m}$. These lines of separation are not distributed all over the chaotic region but are localized in a relatively small region.

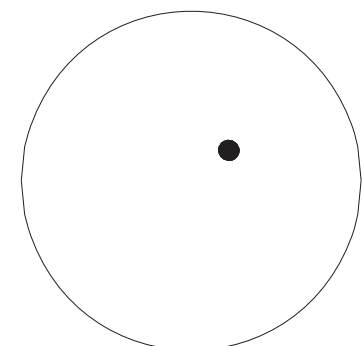

(a)

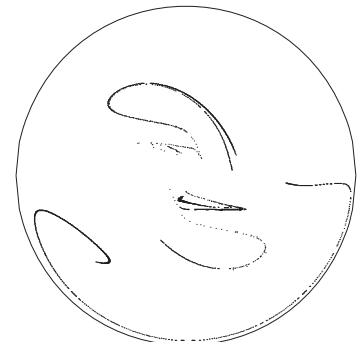

(b)

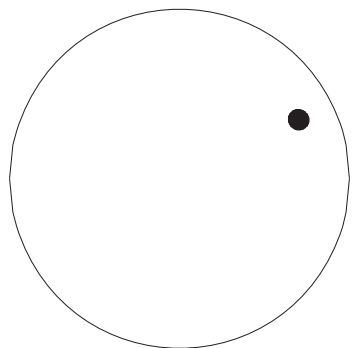

(c)

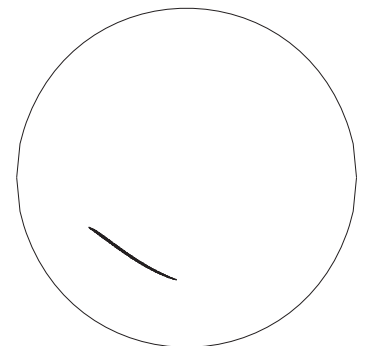

(d)

Figure 18: Cross-sectional movement and deformation of two small blobs shown in (a) and (c). The blobs in (a) and (c) evolve into the configurations shown in (b) and (d) after one period, respectively.

Figure 18 shows the cross-sectional movement and deformation of two small circular blobs that are initially on a cross-section $Z_{m}$, associated with their movement in the axial direction by one period. The blobs that initially include a part of the lines of separation shown in Fig. 17(b), such as the blob in Fig. 18(a), are divided into several components owing to the discontinuity of Poincaré map $M$ on lines of separation and are strongly stretched, as found from Fig. 18(b). On the other hand, the blobs whose initial locations are not close to lines of separation, such as the blob in Fig. 18(c), are stretched only weakly even if they are in chaotic regions, as illustrated in Fig. 18(d). From this result, it is suggested that only the blobs starting from the neighborhood of lines of separation 
are strongly stretched and mixed well in their axial movement by one period. Therefore, the distribution of lines of separation can be used to estimate the efficiency of mixing in a short time (in a few axial periods).

The close correlation between the region where fluid elements are stretched strongly and the region where lines of separation are distributed densely was also shown by Mizuno and Funakoshi (2004).

\section{§5. Concluding remarks}

In Section 2 of the present paper, several studies on Lagrangian chaos, the chaotic motion of fluid particles by two-dimensional time-periodic flows or three-dimensional steady flows, were introduced. There are also studies related to the theory on nearintegrable Hamiltonian systems. For example, we can consider a two-dimensional timeperiodic velocity field of an incompressible fluid that is expressed as the sum of a basic (not necessarily steady) velocity field for which Eq. (1) is integrable in the sense that each fluid particle moves on a time-independent streamline, and a small perturbation velocity that causes the non-integrability of Eq.(1). In such a velocity field, as the magnitude of the perturbation increases from zero, we usually observe in Poincaré sections the breakup of more of the original closed curves that results in the appearance and growth of chaotic regions, whereas remaining closed curves survive as those corresponding to the Kolmogorov-Arnold-Moser (KAM) tori and occupy regular regions. This is the result from the KAM theorem on near-integrable Hamiltonian systems. A few examples of studies related to the KAM theorem and mixing are Wonhas and Vassilicos (2001) on time-periodic vortical flows and Kaper and Wiggins (1993) on slowly-varying timeperiodic flows between eccentric rotating cylinders. For those who are not familiar with the theory on Hamiltonian systems and the KAM theorem, references such as Ottino (1989), Lichtenberg and Lieberman (1992) and Doherty and Ottino (1988) are useful.

In Section 3, studies of chaotic mixing by two-dimensional time-periodic flows or three-dimensional steady flows were reviewed. In addition to these studies, there are a few investigations of chaotic mixing by three-dimensional time-periodic flows. For example, Malyuga et al. (2002) and Speetjens et al. (2006) examined the mixing by a 
three-dimensional Stokes flow in a cylindrical container caused by the periodic motions of cylinder end walls, and Galaktionov et al. (2001) examined the mixing by a similar flow in a cubic cavity. The standard tools used in the theory of dynamical systems are still applicable to such flows. The examination of mixing efficiency based on them is, however, more difficult compared with the cases of two-dimensional time-periodic flows or three-dimensional steady flows, partly because we have to extract information related to mixing from the Poincaré sections defined in a three-dimensional region or from the geometrical structure of one-dimensional or two-dimensional stable and unstable manifolds of hyperbolic periodic points of a Poincaré map in a three-dimensional region, as attempted by Malyuga et al. (2002) and Speetjens et al. (2006). In spite of this difficulty, the studies on the estimation of mixing efficiency by three-dimensional timeperiodic flows are expected to develop in the future, because such flows are common in practical mixing devices. For example, the flow caused by a steady rotation of impellers in a stirred tank is usually three-dimensional and time-periodic, except for the special cases in which flows are steady in the reference frame fixed to the impellers as in the studies by Fountain et al. (1998, 2000).

In Sections 3 and 4, several indices for the estimation of mixing efficiency were introduced and discussed with a special attention to the mixing efficiency in a short time. Besides studies on several indices introduced in these sections, such as the indices related to transport matrices, stable and unstable manifolds of hyperbolic periodic points of Poincaré maps, and lines of separation, there are also interesting studies on various aspects of mixing. An example of such aspects is the asymptotic directionality of fluid elements suggested by Giona et al. $(1999,2000)$ in the study of mixing by two-dimensional time-periodic flows between eccentric rotating cylinders. This property implies the existence of a local asymptotic orientation of fluid elements at each point within chaotic regions. They suggested that information on the asymptotic directionality is useful because it determines the geometrical configuration of unstable manifolds of hyperbolic periodic points of Poincaré maps. This asymptotic directionality was revealed also in dynamo theory by simulations of magnetic fields in fluid flows under a small diffusive effect, in which magnetic field vectors align with the stretching directions (see, for example, Childress and Gilbert, 1995). 
Another interesting aspect of mixing is the relation between the curvature and stretching rate of material lines. From the numerical examination of the time evolution of stretching and curvature of material lines by a two-dimensional time-periodic flow in a lid-driven cavity that yield chaotic motion of fluid particles, Liu and Muzzio (1996) showed that parts of material lines with large curvature are exceptionally compressed by the flow. Similar close correlation between the parts of compression and parts of large curvature was also reported by Hobbs and Muzzio (1998) and Thiffeault (2004). Leonard (2005) also examined a similar problem with the inclusion of torsion of material lines. Other related studies of the relation between the curvature of material lines and the finite-time largest Liapunov exponent in flows that cause the chaotic motion of fluid particles were performed by Tang and Boozer (1996) and Thiffeault and Boozer (2001).

Recently, a measure to estimate the extent of mixing over different length scales called the Mix-Norm was suggested by Mathew et al. (2005). Since the significance of this measure is discussed in a mathematically rigorous way, this study seems to contribute to the development of mathematical theory on mixing.

\section{Acknowledgments}

The author is indebted to the anonymous referees and the editor of this paper for their valuable comments and suggestions during the review process. This work is partly supported by the Grant-in-Aid (No.1430193420) for scientific research and also by the 21st Century COE Program (Center of Excellence for Research and Education on Complex Functional Mechanical Systems) of Ministry of Education, Culture, Sports, Science and Technology.

\section{References}

Anderson, P.D., Galaktionov, O.S., Peters, G.W.M., Meijer, H.E.H., Tucker III, C.L., 2002. Material stretching in laminar mixing flows: extended mapping technique applied to the journal bearing flow. Int. J. Numer. Meth. Fluids 40, 189-196.

Aref, H., 1984. Stirring by chaotic advection. J. Fluid Mech. 143, 1-21.

Aref, H., 1990. Chaotic advection of fluid particles. Phil. Trans. R. Soc. Lond. A 333, 
$273-288$.

Aref, H., 1991. Stochastic particle motion in laminar flows. Phys. Fluids A 3, 10091016.

Aref, H., 2002. The development of chaotic advection. Phys. Fluids 14, 1315-1325.

Aref, H., Jones, S.W., 1989. Enhanced separation of diffusing particles by chaotic advection. Phys. Fluids A 1, 470-474.

Aref, H., Jones, S.W., Mofina, S., Zawadzki, I., 1989. Vortices, kinematics and chaos. Physica D 37, 423-440.

Atobe, T., Funakoshi, M., 1994. Chaotic motion of fluid particles due to the alternate rotations of two eccentric cylinders. J. Phys. Soc. Jpn. 63, 1738-1753.

Atobe, T., Funakoshi, M., Inoue, S., 1995. Orbital instability and chaos in the Stokes flow between two eccentric cylinders. Fluid Dyn. Res. 16, 115-129.

Bajer, K., Moffatt, H.K., 1990. On a class of steady confined Stokes flows with chaotic streamlines. J. Fluid Mech. 212, 337-363.

Ballal, B.Y., Rivlin, R.S., 1977. Flow of a Newtonian fluid between eccentric rotating cylinders: inertial effects. Arch. Rat. Mech. Anal. 62, 237-294.

Beigie, D., Leonard, A., Wiggins, S., 1994. Invariant manifold templates for chaotic advection. Chaos, Solitons Fractals 4, 749-868.

Boyland, P.L., Aref, H., Stremler, M.A., 2000. Topological fluid mechanics of stirring. J. Fluid Mech. 403, 277-304.

Cartwright, J.H.E., Feingold, M., Piro, O., 1999. An introduction to chaotic advection. In: Chaté, H., Villermaux, E., Chomaz, J.-M. (Eds.), Mixing: Chaos and Turbulence, Kluwer Academic/Plenum Publishers, New York, pp.307-342.

Chaiken, J., Chevray, R., Tabor, M., Tan, Q.M., 1986. Experimental study of Lagrangian turbulence in a Stokes flow. Proc. R. Soc. Lond. A 408, 165-174.

Chew, Y.T., Xia, H.M., Shu, C., Wan, S.Y.M., 2005. Techniques to enhance fluid micromixing and chaotic micromixers. Mod. Phys. Lett. B 19, 1567-1570.

Childress, S., Gilbert, A.D., 1995. Stretch, Twist, Fold: the Fast Dynamo. SpringerVerlag, Berlin.

Danckwerts, P.V., 1952. The definition and measurement of some characteristics of mixtures. Appl. Sci. Res. A 3, 279-296. 
Dean, W.R., 1927. Note on the motion of fluid in a curved pipe. Phil. Mag. 4, 208-223. Dean, W.R., 1928. The streamline motion of fluid in a curved pipe. Phil. Mag. 5, 673-693.

Dittrich, P.S., Tachikawa, K., Manz, A., 2006. Micro total analysis systems. Latest advancements and trends. Anal. Chem. 78, 3887-3907.

Doherty, M.F., Ottino, J.M., 1988. Chaos in deterministic systems: strange attractors, turbulence, and applications in chemical engineering. Chem. Eng. Sci. 43, 139-183.

Dombre, T., Frisch, U., Greene, J.M., Hénon, M., Mehr, A., Soward, A.M., 1986. Chaotic streamlines in the ABC flows. J. Fluid Mech. 167, 353-391.

Dutta, P., Chevray, R., 1995. Inertial effects in chaotic mixing with diffusion. J. Fluid Mech. 285, 1-16.

Finn, M.D., Cox, S.M., 2001. Stokes flow in a mixer with changing geometry. J. Eng. Math. 41, 75-99.

Finn, M.D., Cox, S.M., Byrne, H.M., 2004. Mixing measures for a two-dimensional chaotic Stokes flow. J. Eng. Math. 48, 129-155.

Fountain, G.O., Khakhar, D.V., Ottino, J.M., 1998. Visualization of three-dimensional chaos. Science 281, 683-686.

Fountain, G.O., Khakhar, D.V., Mezić, I., Ottino, J.M., 2000. Chaotic mixing in a bounded three-dimensional flow. .J. Fluid Mech. 417, 265-301.

Franjione, J.G., Leong, C.-W., Ottino, J.M., 1989. Symmetries within chaos: A route to effective mixing. Phys. Fluids A 1, 1772-1783.

Funakoshi, M., 2001. Lagrangian chaos and mixing of fluids. Japan J. Indust. Appl. Math. 18, 613-626.

Galaktionov, O.S., Anderson, P.D., Kruijt, P.G.M., Peters, G.W.M., Meijer, H.E.H., 2001. A mapping approach for three-dimensional distributive mixing analysis. Computers and Fluids 30, 271-289.

Galaktionov, O.S., Anderson, P.D., Peters, G.W.M., Meijer, H.E.H., 2003. Analysis and optimization of Kenics static mixers. Int. Polymer Processing 18, 138-150.

Giona, M., Adrover, A., Muzzio, F.J., Cerbelli, S., Alvarez, M.M., 1999. The geometry of mixing in time-periodic chaotic flows. I. Asymptotic directionality in physically realizable flows and global invariant properties. Physica D 132, 298-324. 
Giona, M., Adrover, A., Muzzio, F.J., Cerbelli, S., 2000. The geometry of mixing in 2-d time-periodic chaotic flows. Chem. Eng. Sci. 55, 381-389.

Hardt, S., Pennemann, H., Schönfeld, F., 2006. Theoretical and experimental characterization of a low-Reynolds number split-and-recombine mixer. Microfluid Nanofluid 2, $237-248$.

Hessel, V., Löwe, H., Schönfeld, F., 2005. Micromixers - a review on passive and active mixing principles. Chem. Eng. Sci. 60, 2479-2501.

Hobbs, D.M., Muzzio, F.J., 1998. The curvature of material lines in a three-dimensional chaotic flow. Phys. Fluids 10, 1942-1952.

Ichida, Y., Funakoshi, M., 1998. Chaotic mixing under the flow between two eccentric cylinders, Res. Inst. Math. Sci., Kyoto Univ., Kokyuroku No.1029, 31-52 (in Japanese). Ishii, K., Iwatsu, R., 1989. Numerical simulation of the Lagrangian flow structure in a driven-cavity. In: Moffatt, H.K., Tsinobar, A. (Eds.), Topological Fluid Mechanics, Cambridge University Press, Cambridge, pp.54-63.

Iwatsu, R., Ishii, K., Kawamura, T., Kuwahara, K., Hyun, J.M., 1989. Simulation of transition to turbulence in a cubic cavity. AIAA Pap. No.89-0040.

Jeffery, G.B., 1922. The rotation of two circular cylinders in a viscous fluid. Proc. R. Soc. Lond. A 101, 169-174.

Jones, S.W., Aref, H., 1988. Chaotic advection in pulsed source-sink systems. Phys. Fluids 31, 469-485.

Jones, S.W., Thomas, O.M., Aref, H., 1989. Chaotic advection by laminar flow in a twisted pipe. J. Fluid Mech. 209, 335-357.

Kaper, T.J., Wiggins, S., 1993. An analytical study of transport in Stokes flows exhibiting large-scale chaos in the eccentric journal bearing. J. Fluid Mech. 253, 211-243.

Kawazoe, H., 2004. Mixing and deformation of fluid elements by the flow between two eccentric cylinders. Master thesis, Graduate School of Informatics, Kyoto Univ.

Kawazoe, H., Funakoshi, M., Kaneko, Y., 2004. Indices of the mixing efficiency in a short time by a flow between two eccentric cylinders. Res. Inst. Math. Sci., Kyoto Univ., Kokyuroku No.1406, 62-79 (in Japanese).

Khakhar, D.V., Franjione, J.G., Ottino, J.M., 1987. A case study of chaotic mixing in deterministic flows: the partitioned-pipe mixer. Chem. Eng. Sci. 42, 2909-2926. 
King, G.P., Rowlands, G., Rudman, M., Yannacopoulos, A.N., 2001. Predicting chaotic dispersion with Eulerian symmetry measures: Wavy Taylor-vortex flow. Phys. Fluids $13,2522-2528$.

Kruijt, P.G.M., Galaktionov, O.S., Anderson, P.D., Peters, G.W.M., Meijer, H.E.H., 2001. Analyzing mixing in periodic flows by distribution matrices: mapping method. AIChE J. 47, 1005-1015.

Kusch, H.A., Ottino, J.M., 1992. Experiments on mixing in continuous chaotic flows. J. Fluid Mech. 236, 319-348.

Leonard, A., 2005. Curvature and torsion of material lines in chaotic flows. Fluid Dyn. Res. 36, 261-275.

Leong, C.W., Ottino, J.M., 1989. Experiments on mixing due to chaotic advection in a cavity. J. Fluid Mech. 209, 463-499.

Lichtenberg, A.J., Lieberman, M.A., 1992. Regular and Chaotic Dynamics, 2nd ed. Springer-Verlag, New York.

Ling, F.H., 1993. Chaotic mixing in a spatially periodic continuous mixer. Phys. Fluids A 5, 2147-2160.

Liu, M., Muzzio, F.J., 1996. The curvature of material lines in chaotic cavity flows. Phys. Fluids 8, 75-83.

Malyuga, V.S., Meleshko, V.V., Speetjens, M.F.M., Clercx, H.J.H., van Heijst, G..J.F., 2002. Mixing in the Stokes flow in a cylindrical container. Proc. R. Soc. Lond. A 458, 1867-1885.

Mandelbrot, B.B., 1982. The Fractal Geometry of Nature. W. H. Freeman, New York. Mathew, G., Mezić, I., Petzold, L., 2005. A multiscale measure for mixing. Physica D $211,23-46$.

Meleshko, V.V., Galaktionov, O.S., Peters, G.W.M., Meijer, H.E.H., 1999. Threedimensional mixing in Stokes flow: the partitioned pipe mixer problem revisited. Eur. J. Mech. B/Fluids 18, 783-792.

Mizuno, Y., Funakoshi, M., 2002. Chaotic mixing due to a spatially periodic threedimensional flow. Fluid Dyn. Res. 31, 129-149.

Mizuno, Y., Funakoshi, M., 2004. Chaotic mixing caused by an axially periodic steady flow in a partitioned-pipe mixer. Fluid Dyn. Res. 35, 205-227. 
Mizuno, Y., Funakoshi, M., 2005. Reynolds number dependences of velocity field and fluid mixing in partitioned-pipe mixer. J. Phys. Soc. Jpn. 74, 1479-1489.

Muzzio, F.J., Swanson, P.D., Ottino, J.M., 1992. Mixing distributions produced by multiplicative stretching in chaotic flows. Int. J. Bifurcations and Chaos 2, 37-50.

Ottino, J.M., 1989. The Kinematics of Mixing: Stretching, Chaos, and Transport. Cambridge University Press, Cambridge.

Ottino, J.M., 1990. Mixing, chaotic advection, and turbulence. Annu. Rev. Fluid Mech. $22,207-253$.

Ottino, J.M., Shinbrot, T., 1999. Comparing extremes: mixing of fluids, mixing of solids. In: Chaté, H., Villermaux, E., Chomaz, J.-M. (Eds.), Mixing: Chaos and Turbulence, Kluwer Academic/Plenum Publishers, New York, pp.163-186.

Ottino, J.M., Wiggins, S., 2004. Introduction: mixing in microfluidics. Phil. Trans. R. Soc. Lond. A 362, 923-935.

Rom-Kedar, V., I.eonard, A., Wiggins, S., 1990. An analytical study of transport, mixing and chaos in an unsteady vortical flow. J. Fluid Mech. 214, 347-394.

Speetjens, M.F.M., Clercx, H.J.H., van Heijst, G.J.F., 2006. Inertia-induced coherent structures in a time-periodic viscous mixing flow. Phys. Fluids 18, 083603.

Spencer, R.S., Wiley, R.M., 1951. The mixing of very viscous liquids. J. Colloid Sci. 6, $133-145$.

Stremler, M.A., Haselton, F.R., Aref, H., 2004. Designing for chaos: applications of chaotic advection at the microscale. Phil. Trans. R. Soc. Lond. A 362, 1019-1036.

Sturman, R., Ottino, J.M., Wiggins, S., 2006. The Mathematical Foundations of Mixing - The Linked Twist Map as a Paradigm in Applications: Micro to Macro, Fluids to Solids-. Cambridge University Press, Cambridge.

Swanson, P.D., Ottino, J.M., 1990. A comparative computational and experimental study of chaotic mixing of viscous fluids. J. Fluid Mech. 213, 227-249.

Tang, X.Z., Boozer, A.H., 1996. Finite time Lyapunov exponent and advection-diffusion equation. Physica D 95, 283-305.

Thiffeault, J.-L., 2004. Stretching and curvature of material lines in chaotic flows. Physica D 198, 169-181.

Thiffeault, J.-L., Boozer, A.H., 2001. Geometrical constraints on finite-time Lyapunov 
exponents in two and three dimensions. Chaos 11, 16-28.

Thiffeault, J.-L., Finn, M.D., 2006. Topology, braids and mixing in fluids. Phil. Trans. R. Soc. A 364, 3251-3266.

Wiggins, S., 1992. Chaotic Transport in Dynamical Systems. Springer-Verlag, New York.

Wiggins, S., Ottino, J.M., 2004. Foundations of chaotic mixing. Phil. Trans. R. Soc. Lond. A 362, 937-970.

Wilson, M.C.T., Summers, J.L., Kapur, N., Gaskell, P.H., 2006. Stirring and transport enhancement in a continuously modulated free-surface flow. J. Fluid Mech. 565, 319351.

Wonhas, A., Vassilicos, J.C., 2001. Mixing in frozen and time-periodic two-dimensional vortical flows. J. Fluid Mech. 442, 359-385. 